\title{
Multistatic Synthetic Aperture Radar Image Formation
}

\author{
V. Krishnan, J. Swoboda, Member, IEEE, C. E. Yarman, Member, IEEE, and B. Yazici, Senior Member, IEEE
}

\begin{abstract}
In this paper, we consider a multistatic synthetic aperture radar (SAR) imaging scenario where a swarm of airborne antennas, some of which are transmitting, receiving or both, are traversing arbitrary flight trajectories and transmitting arbitrary waveforms without any form of multiplexing. The received signal at each receiving antenna may be interfered by the scattered signal due to multiple transmitters and additive thermal noise at the receiver. In this scenario, standard bistatic SAR image reconstruction algorithms result in artifacts in reconstructed images due to these interferences. In this paper, we use microlocal analysis in a statistical setting to develop a filtered-backprojection (FBP) type analytic image formation method that suppresses artifacts due to interference while preserving the location and orientation of edges of the scene in the reconstructed image. Our FBP-type algorithm exploits the second-order statistics of the target and noise to suppress the artifacts due to interference in a mean-square sense. We present numerical simulations to demonstrate the performance of our multistatic SAR image formation algorithm with the FBP-type bistatic SAR image reconstruction algorithm. While we mainly focus on radar applications, our image formation method is also applicable to other problems arising in fields such as acoustic, geophysical and medical imaging.
\end{abstract}

Index Terms-Filtered-backprojection, microlocal analysis, multistatic, radar, synthetic aperture imagery (SAR).

\section{INTRODUCTION}

$\mathbf{I}$ $\mathrm{N}$ synthetic aperture radar (SAR) imaging, a scene of interest is illuminated by electromagnetic waves transmitted from an antenna mounted on an airborne platform. The aim is to reconstruct an image of the scene from the measurement of the scattered waves.

In monostatic SAR, the transmitter and receiver antennas are co-located. In bistatic SAR, the transmitter and receiver antennas are located on sufficiently far-apart platforms [32]. In multistatic SAR, which is the focus of this paper, multiple trans-

Manuscript received May 08, 2009; revised November 24, 2009. First published December 31, 2009; current version published April 16, 2010. This work was supported in part by the Air Force Office of Scientific Research (AFOSR) under the agreement FA9550-07-1-0363 and in part by the National Science Foundation (NSF) under Grant CCF-08030672. The associate editor coordinating the review of this manuscript and approving it for publication was Dr. Brian D. Rigling.

V. Krishnan and B. Yazici are with the Department of Electrical, Computer and Systems Engineering, Rensselaer Polytechnic Institute, Troy, NY 12180 USA (e-mail: krishv2@rpi.edu; yazici@ecse.rpi.edu).

J. Swoboda is with the MITRE Corporation, Bedford, MA 01730-1420 USA (e-mail: jswoboda@mitre.org).

C. E. Yarman is with the Houston Technology Center, WesternGeco-Schlumberger, Houston, TX 77042 USA (e-mail: yarman@ecse.rpi.edu)

Color versions of one or more of the figures in this paper are available online at http://ieeexplore.ieee.org.

Digital Object Identifier 10.1109/TIP.2009.2039662 mitter and receiver antennas are used to image a scene. Multistatic SAR offers a variety of potential gains in scene information. The availability of multiple illumination and scattered measurements from different perspectives has the potential for improved overall resolution when the scattered measurements are fused [7]. Also, some of the electronic countermeasures that have been devised for monostatic radar are less effective against distributed radar systems [9], [13]. Finally, multistatic measurements can provide a better ability to distinguish targets from clutter [31].

Both bistatic and multistatic radar systems have received increased attention in recent years. In [24], using multiple bistatic images, algorithms that generalize monostatic interferometric processing and stereo SAR to reconstruct 3-D surfaces are given. In [4], a multistatic ambiguity function was recently proposed for a system with a single transmitter and multiple receivers, and in [3] for multiple transmitters and receivers. An autofocus algorithm for multistatic SAR systems using multiple transmitters was presented in [17]. In [28], a multistatic SAR image formation method based on time-frequency filtering and image combination was presented for a system consisting of a single transmitter and multiple receivers. The impact of oscillator noise in multistatic SAR systems was considered in [16].

In many multistatic radar systems, it is assumed that each receiver can decompose the received signal into components due to each transmitting antenna. This can be achieved by separating the transmitted signals by some form of multiplexing in time, frequency or coding which requires central coordination of the transmit signal parameters. In this case, the multistatic synthetic aperture image formation problem reduces to the bistatic synthetic aperture image formation problem which has been well-studied [2], [6], [23], [25], [26], [33]. For bistatic SAR image reconstruction algorithms involving antennas that can form narrow beam and traversing linear or circular trajectories, see [2], [23], [25], and [26]. For bistatic SAR image reconstruction algorithms involving antennas with poor directivity and traversing arbitrary flight trajectories, see [6] and [33].

In this paper, we consider a multistatic SAR system where several transmitters and receivers are employed in imaging a scene and that their trajectories and waveforms are arbitrary but known. We assume that the receivers cannot decompose the received signal according to which transmitter emitted the signal. A simple multistatic SAR scenario with two transmitters and a receiver is illustrated in Fig. 1. Such a scenario emerges when the illumination is provided by sources of opportunity, such as communication satellites or cell-phone towers or when the central coordination of a swarm of antennas deployed on uninhab- 


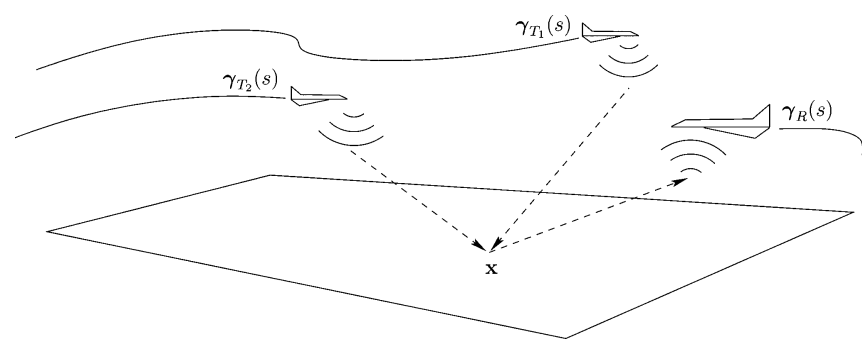

Fig. 1. Multistatic SAR geometry with two transmitters and a single receiver.

ited aerial vehicles (UAVs) is either not possible or desirable. Bistatic reconstruction algorithms, given for example in [2] and [33], are not suited for this scenario, since they are designed for a given transmitter-receiver measurement pair and, therefore, result in artifacts in the reconstructed image due to interference caused by multiple transmitters. Fig. 2 shows the artifacts induced when we use the bistatic reconstruction algorithm of [33]. In this example, the multistatic SAR geometry involves two transmitters and a receiver traversing in a circular trajectory over the scene shown in Fig. 2(a) with a phase difference of $\pi / 3$.

In this paper, we introduce a filtered-backprojection type reconstruction algorithm for multistatic SAR with multiple transmitters transmitting arbitrary waveforms. For the ease of exposition, our reconstruction algorithm assumes that the target to be imaged is composed of isotropic scatterers. In Section V, we generalize our algorithm to take into account anisotropic scattering. We use microlocal analysis [10], [11] to develop an approximate analytic image reconstruction method. Microlocal techniques lead to inversion methods that have the desirable property that visible edges in the scene will appear in the reconstructed image at the correct location and orientation. They can also account for various factors such as arbitrary flight trajectory, nonflat ground topography, antenna beam pattern, transmitted waveforms and geometric spreading. Furthermore, microlocal techniques give rise to FBP-type inversion, which is a direct (noniterative) method that can be implemented efficiently by fast backprojection techniques [19].

Our method involves backprojecting the received data with respect to each transmitter while suppressing the interference caused by other transmitters via a suitably designed filter. We assume that we have a priori knowledge of the second-order statistics of the scene to be reconstructed. We determine the secondorder statistics of the artifacts caused by interference based on antenna trajectories and design a filter that preserves the edges in the received data due to the scene while suppressing the artifacts induced by the interfering transmitters in the mean-square sense. We form the final image by the coherent superposition of the images reconstructed using all transmitter-receiver pairs.

While we primarily focus on SAR imaging, the multistatic imaging method introduced in this paper can be also applied to synthetic aperture imaging problems in acoustics, geophysics and medical imaging. Additionally, the ideas introduced here can be extended to develop autofocus methods for multistatic SAR and for the suppression of artifacts in multipathing environments.
TABLE I

NOTATION

\begin{tabular}{cl}
\hline \hline Symbol & \multicolumn{1}{c}{ Description } \\
\hline$T$ & Scene reflectivity function \\
$s$ & slow-time \\
$t$ & fast-time \\
$p$ & Index for the $p^{t h}$ transmitter \\
$q$ & Index for the $q^{t h}$ receiver \\
$M$ & Number of transmitters \\
$N$ & Number of receivers \\
$\gamma_{T_{p}}(s)$ & Path of $p^{t h}$ transmitter \\
$\gamma_{R_{q}}(s)$ & Path of $q^{t h}$ receiver \\
$n_{q}$ & Additive noise at the $q^{t h}$ receiver \\
$d_{p q}$ & Ideal bi-static received signal at the $q^{t h}$ receiver \\
& due to the $p^{t h}$ transmitter \\
$d_{q}$ & Ideal multi-static received signal at the $q^{t h}$ receiver \\
$R_{T}$ & Autocorrelation function of $T$ \\
$S_{T}$ & Power spectral density function of $T$ \\
$R_{n_{q}}$ & Autocorrelation function of $n_{q}$ \\
$S_{n_{q}}$ & Power spectral density function of additive noise \\
& at the $q^{t h}$ receiver \\
$\mathcal{F}_{p q}$ & Expectation operator \\
$\mathcal{F}_{q}$ & Bi-static SAR forward model for the ideal data at the $q^{t h}$ \\
& receiver due to the $p^{t h}$ transmitter \\
$\mathcal{K}_{p q}$ & Multi-static forward model for the ideal data \\
& Filtered-backprojection operator of data at the $q^{t h}$ receiver \\
with respect to the $p^{t h}$ transmitter
\end{tabular}

This paper is organized as follows. In Section II, we introduce the multistatic SAR forward model and in Section III, we discuss bistatic SAR image formation and in Section IV, we explain in detail the idea behind the filtered-backprojection method for multistatic SAR and derive the filter. In Section V, we state the modifications of our reconstruction method to incorporate anisotropic scattering. The numerical simulations are presented in Sections VI and VII concludes the paper. The stationary phase method is stated in Appendix A and the derivations based on which the filters in Section IV are derived, are worked out in Appendixes B-E.

\section{Forward Model For Multistatic Synthetic APERTURE RADAR DATA}

\section{Notation}

We use the following notational conventions in this paper. Operators are denoted in calligraphic font, points in $\mathbb{R}^{n}$ for $n \geq$ $3, \mathbb{R}^{2}$ and $\mathbb{R}$ are denoted in bold Roman, bold italic and italic letters respectively. Table I includes all other notation used in the paper.

\section{A. Deterministic Forward Model}

In this subsection, we give the multistatic SAR forward model in a deterministic setting. The deterministic setting assumes that the received signal is noise-free and clutter-free and that the target reflectivity function $T$ is deterministic. The 


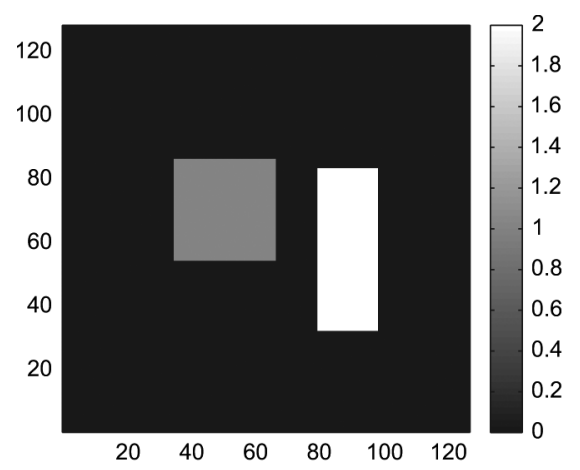

(a)

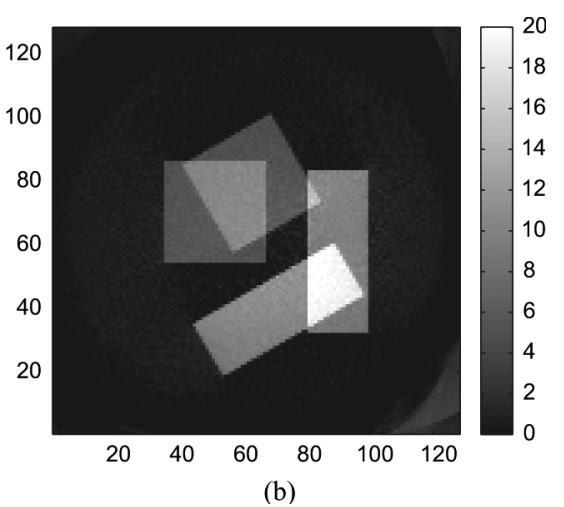

(b)

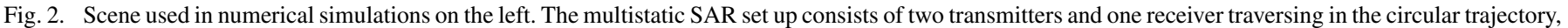

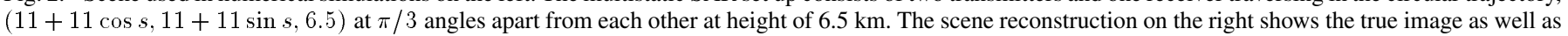
artifacts when the data at the receiver is backprojected with respect to the first transmitter using the bistatic FBP algorithm of [33].

forward model presented here generalizes the forward models of monostatic SAR [21], [22] and bistatic SAR [33].

We consider a multistatic SAR setup where there are $M$ transmitters and $N$ receivers with $M \geq 2$ and $N \geq 1$. We index the transmitters by $T_{p}$ for $p=1, \ldots, M$ and receivers by $R_{q}$ for $q=1, \ldots, N$. Let $\boldsymbol{\gamma}_{T_{p}}(s)$ and $\boldsymbol{\gamma}_{R_{q}}(s)$ for $s \in\left[s_{0}, s_{1}\right]$ be the trajectories of the transmitters and the receivers respectively. Let $\psi: \mathbb{R}^{2} \rightarrow \mathbb{R}$ be a smooth function representing the ground topography. That is, the ground topography is given by $\mathbf{x}=(\boldsymbol{x}, \psi(\boldsymbol{x}))$ for $\boldsymbol{x} \in \mathbb{R}^{2}$. We assume that the electromagnetic waves propagate in free-space and then scatter in a thin region near the earth's surface. Consequently, we assume that the target reflectivity function $T$, is a function of the variable $\boldsymbol{x} \in \mathbb{R}^{2}$ [21]. Under the start-stop approximation and the single scattering Born approximation [22], the ideal received signal $d_{p q}$ at the $q$ th receiver due to the $p$ th transmitter is given by [33]

$$
\begin{aligned}
d_{p q}(s, t)= & \mathcal{F}_{p q}[T](s, t) \\
:= & \int e^{-\mathrm{i} \omega\left(t-1 / c_{0} R_{p q}(s, \boldsymbol{x})\right)} \\
& \times A_{p q}(\boldsymbol{x}, s, \omega) T(\boldsymbol{x}) \mathrm{d} \boldsymbol{x} \mathrm{d} \omega
\end{aligned}
$$

where $s \in\left[s_{0}, s_{1}\right]$ is the slow-time variable, $t \in\left[t_{0}, t_{1}\right]$ is the fast-time variable. Such a distinction is made, because the speed of the airborne platforms carrying transmitters and receivers is much lower than the speed of light. In (1)

$$
R_{p q}(s, \boldsymbol{x})=\left|\mathbf{x}-\boldsymbol{\gamma}_{T_{p}}(s)\right|+\left|\mathbf{x}-\gamma_{R_{q}}(s)\right|
$$

is the total distance from the transmitter position $\boldsymbol{\gamma}_{T_{p}}(s)$ to $\mathbf{X}$ and from $\mathbf{x}$ to the receiver position $\gamma_{R_{q}}(s), c_{0}$ is the speed of light in free-space, $\omega$ is the temporal frequency and $A_{p q}(s, \boldsymbol{x}, \omega)$ is a function that takes into account the transmitter and receiver antenna beam patterns, the transmitted waveforms and geometrical spreading factors [5], [20], [21]. We assume that the functions $A_{p q}(s, \boldsymbol{x}, \omega)$ for $p=1, \ldots, M$ and $q=1, \ldots, N$ satisfy the following estimate for some real number $m_{p q}$

$$
\left|\partial_{\omega}^{\alpha} \partial_{s}^{\beta} \partial_{x_{1}}^{\rho_{1}} \partial_{x_{2}}^{\rho_{2}} A_{p q}(\boldsymbol{x}, s, \omega)\right| \leq C\left(1+|\omega|^{2}\right)^{\left(m_{p q}-|\alpha|\right) / 2}
$$

for $(s, \boldsymbol{x}) \in K$ and $\omega \in \mathbb{R}$. Here $K \subset \mathbb{R} \times \mathbb{R}^{2}$ is any compact set and $\alpha$ and $\beta, \rho_{1}, \rho_{2}$ are any non-negative integers, and $C$ is a constant in terms of $p, q, K, \alpha, \beta, \rho_{1}, \rho_{2}$. These estimates are satisfied when the antenna is broadband and when the source waveform is a band limited waveform. These assumptions are needed to make various stationary phase calculations. Furthermore, these assumptions make each operator $\mathcal{F}_{p q}$ a Fourier Integral Operator [8], [29], [30].

The bistatic SAR image formation problem involves reconstruction of the target reflectivity function $T$ from measurements $d_{p q}(s, t)$, for $s \in\left[s_{0}, s_{1}\right], t \in\left[t_{0}, t_{1}\right]$ and each $p$ and $q$ based on the model (1).

For the multistatic SAR case, we assume that the ideal received signal $d_{q}$ at the $q$ th receiver is

$$
\begin{aligned}
d_{q}(s, t)= & \mathcal{F}_{q}[T](s, t):=\sum_{p=1}^{M} \mathcal{F}_{p q}[T](s, t) \\
= & \sum_{p=1}^{M} \int e^{-\mathrm{i} \omega\left(t-1 / c_{0} R_{p q}(s, \boldsymbol{x})\right)} \\
& \times A_{p q}(\boldsymbol{x}, s, \omega) T(\boldsymbol{x}) \mathrm{d} \boldsymbol{x} \mathrm{d} \omega .
\end{aligned}
$$

Furthermore, we assume that the multistatic received data $d_{q}$ at the $q$ th receiver is not necessarily decomposable into components $d_{p q}, p=1, \ldots, M$. Thus, the multistatic SAR image formation problem involves reconstruction of the target reflectivity function $T$ using the data $d_{q}(s, t)$ for $s \in\left[s_{0}, s_{1}\right], t \in\left[t_{0}, t_{1}\right]$ and $q=1, \ldots, N$ based on the model (5).

\section{B. Statistical Forward Model}

In this section, we model the target reflectivity as a random field and take into account the additive thermal noise at each receiver to model the multistatic measurements. These considerations result in a forward model defined in a statistical setting. We use this statistical forward model in developing a multistatic image formation method presented in the following section. In the presence of thermal noise and random target reflectivity function, we consider the following model for the measurement at the $q$ th receiver

$$
d_{q}(s, t)=\mathcal{F}_{q}[T](s, t)+n_{q}(s, t)
$$

where $n_{q}$ denotes the thermal noise at the $q$ th receiver and $\mathcal{F}_{q}$ is defined as in (5). 
Without loss of generality, we assume that both $T$ and $n_{q}$ for $q=1, \ldots, N$ are zero-mean random processes. That is, denoting $\mathrm{E}$ as the expectation operator, we have

$$
\begin{aligned}
\mathrm{E}[T(\boldsymbol{x})] & =0 \text { for } \boldsymbol{x} \in \mathbb{R}^{2} \\
\mathrm{E}\left[n_{q}(s, t)\right] & =0 \text { for }(s, t) \in\left[s_{0}, s_{1}\right] \times\left[t_{0}, t_{1}\right] \text { for all } q .
\end{aligned}
$$

We also assume that $T$ is stationary, and $n_{q}$ is statistically uncorrelated in slow-time variable $s$ and stationary in fast-time variable $t$. To state these assumptions more precisely, we first define the autocovariance functions, $R_{T}$ and $R_{n_{q}}$, of $T$ and $n_{q}$, respectively

$$
\begin{aligned}
R_{T}\left(\boldsymbol{x}, \boldsymbol{x}^{\prime}\right) & =\mathrm{E}\left[T(\boldsymbol{x}) \overline{T\left(\boldsymbol{x}^{\prime}\right)}\right] \text { for } \boldsymbol{x}, \boldsymbol{x}^{\prime} \in \mathbb{R}^{2} \\
R_{n_{q}}\left(s, t, s^{\prime}, t^{\prime}\right) & =\mathrm{E}\left[n_{q}(s, t) \overline{n_{q}\left(s^{\prime}, t^{\prime}\right)}\right]
\end{aligned}
$$

for $(s, t) \in\left[s_{0}, s_{1}\right] \times\left[t_{0}, t_{1}\right]$ and $q=1, \ldots, N$. We next define

$$
\widetilde{S}_{T}\left(\boldsymbol{\zeta}, \boldsymbol{\zeta}^{\prime}\right)=\frac{1}{(2 \pi)^{4}} \int e^{\mathrm{i}\left(\boldsymbol{x} \cdot \boldsymbol{\zeta}-\boldsymbol{x}^{\prime} \cdot \boldsymbol{\zeta}^{\prime}\right)} R_{T}\left(\boldsymbol{x}, \boldsymbol{x}^{\prime}\right) \mathrm{d} \boldsymbol{x} \mathrm{d} \boldsymbol{x}^{\prime} .
$$

Under the stationarity assumption, the power spectral density function $S_{T}$ of $T$ satisfies

$$
\tilde{S}_{T}\left(\boldsymbol{\zeta}, \boldsymbol{\zeta}^{\prime}\right)=S_{T}(\boldsymbol{\zeta}) \delta\left(\boldsymbol{\zeta}-\boldsymbol{\zeta}^{\prime}\right)
$$

Similarly, we define

$$
\widetilde{S}_{n_{q}}\left(s, \omega, s^{\prime}, \omega^{\prime}\right)=\frac{1}{(2 \pi)^{2}} \int e^{\mathrm{i}\left(\omega t-\omega^{\prime} t^{\prime}\right)} R_{n_{q}}\left(s, t, s^{\prime}, t^{\prime}\right) \mathrm{d} t \mathrm{~d} t^{\prime} .
$$

Under the assumptions that $n_{q}$ is stationary in $t$ and statistically uncorrelated in $s$, the power spectral density function $S_{n_{q}}$ of $n_{q}$ satisfies

$$
\tilde{S}_{n_{q}}\left(s, \omega, s^{\prime}, \omega^{\prime}\right)=S_{n_{q}}(\omega, s) \delta\left(s-s^{\prime}\right) \delta\left(\omega-\omega^{\prime}\right) .
$$

Finally, we assume that the random processes $T$ and $n_{q}$ for $q=$ $1, \ldots, N$ are statistically uncorrelated. This implies

$$
\mathrm{E}\left[T(\boldsymbol{x}) n_{q}(s, t)\right]=0
$$

for all $\boldsymbol{x} \in \mathbb{R}^{2},(s, t) \in\left[s_{0}, s_{1}\right] \times\left[t_{0}, t_{1}\right]$ and $q=1, \ldots, N$.

In summary, the multistatic data model that we use in this paper is given by

$$
d_{q}(s, t)=\sum_{p=1}^{M} \mathcal{F}_{p q}[T](s, t)+n_{q}(s, t) \quad q=1, \ldots, N
$$

where $\mathcal{F}_{p q}$ are the forward operators for the ideal bistatic measurements defined in (1); $T$ and $n_{q}$ are random processes satisfying the statistical properties stated in this subsection and all integrals in $\mathcal{F}_{p q}$ are understood in the mean-square sense.

\section{BISTATIC IMAGE FORMATION}

The filtered-backprojection methods introduced in [21], [34], and [33] provide a powerful approach to the monostatic and bistatic SAR image formation. This approach is very versatile and has a number of advantages: The resulting methods are direct and noniterative. As a result, they can be made computationally efficient [19]. They can be applied to nonideal image acquisition scenarios involving arbitrary flight trajectories, arbitrary transmitter and receiver waveforms, nonflat ground topography, and noise and clutter [34]. While filtered-backprojection methods give rise to approximate reconstruction formulas, in ideal scenarios these inversion formulas often become exact. For instance, the formulas described in [21], [34] become exact inversion formulas for a broad-band antenna moving along a straight flight trajectory above a flat topography [1]. Furthermore, the filtered-backprojection techniques reconstruct the visible edges of the target reflectivity function at the right location and right orientation. Thus, they are edge preserving.

These desirable features motivate the development of a filtered-backprojection method for the multistatic SAR image formation problem. We first briefly summarize the filter derived for the bistatic image formation problem in [33]. Recall that in bistatic SAR, the ideal received signal at the $q$ th receiver due to the $p$ th transmitter is modeled by $d_{p q}$ in (1). The filtered-backprojection operator for bistatic SAR is

$$
\begin{aligned}
\hat{T}_{p q}(\boldsymbol{z}):= & \mathcal{K}_{p q} d_{p q}(z) \\
= & \int e^{\mathrm{i} \omega\left(t-1 / c_{0} R_{p q}(s, \boldsymbol{z})\right)} \\
& \times B_{p q}(\boldsymbol{z}, s, \omega) d_{p q}(s, t) \mathrm{d} s \mathrm{~d} t \mathrm{~d} \omega .
\end{aligned}
$$

where $B_{p q}$ is the filter to be determined. We denote $\hat{T}_{p q}(z)$ to be the image formed.

Substituting the bistatic data model (1) into (17), we obtain

$$
\begin{aligned}
\hat{T}_{p q}(\boldsymbol{z})=\int e^{\mathrm{i} \omega / c_{0}\left(R_{p q}(s, \boldsymbol{x})-R_{p q}(s, \boldsymbol{z})\right)} B_{p q}(\boldsymbol{z}, s, \omega) \\
\\
\times A_{p q}(\boldsymbol{x}, s, \omega) T(\boldsymbol{x}) \mathrm{d} \boldsymbol{x} \mathrm{d} s \mathrm{~d} \omega .
\end{aligned}
$$

We write

$$
R_{p q}(s, \boldsymbol{x})-R_{p q}(s, \boldsymbol{z})=(\boldsymbol{x}-\boldsymbol{z}) \cdot \Sigma_{p q}(s, \boldsymbol{x}, \boldsymbol{z})
$$

where

$$
\Sigma_{p q}(s, \boldsymbol{x}, \boldsymbol{z})=\int_{0}^{1} \nabla R_{p q}(s, \boldsymbol{z}+\lambda(\boldsymbol{x}-\boldsymbol{z})) \mathrm{d} \lambda .
$$

Here $\nabla$ is the gradient operator in the $x$ variable. For $x=z$

$$
\Sigma_{p q}(s, \boldsymbol{z}, \boldsymbol{z})=\left[D\left(\boldsymbol{z}_{1}, \boldsymbol{z}_{2}\right)\right]^{T}\left(\mathbf{z}-\widehat{\boldsymbol{\gamma}_{T_{p}}}(s)+\mathbf{z}-\widehat{\boldsymbol{\gamma}_{R_{q}}}(s)\right)
$$

with

$$
D\left(z_{1}, z_{2}\right)=\left(\begin{array}{cc}
1 & 0 \\
0 & 1 \\
\frac{\partial \psi}{\partial z_{1}} & \frac{\partial \psi}{\partial \boldsymbol{z}_{2}}
\end{array}\right)
$$

Here, the hat notation over a vector denotes the unit vector in that direction.

In other words, $\Sigma_{p q}(s, z, z)$ is the projection of the bisector of the unit vectors $\mathbf{z}-\boldsymbol{\gamma}_{T_{p}}(s)$ and $\mathbf{z}-\widehat{\gamma_{R_{q}}}(s)$ onto the tangent 
plane of the ground topography at $z$. Now, making the change of variables

$$
(s, \omega) \rightarrow \boldsymbol{\xi}=\frac{\omega}{c_{0}} \Sigma_{p q}(s, \boldsymbol{x}, \boldsymbol{z})
$$

in the integral (17) and letting $\hat{T}_{p q}(z)$ be the image formed, we obtain

$$
\begin{aligned}
\hat{T}_{p q}(\boldsymbol{z})=\int \mathrm{e}^{\mathrm{i}(\boldsymbol{x}-\boldsymbol{z}) \cdot \boldsymbol{\xi}} B_{p q}(\boldsymbol{z}, \boldsymbol{\xi}) A_{p q}(\boldsymbol{x}, \boldsymbol{\xi}) & \\
& \times\left|J_{p q}(\boldsymbol{x}, \boldsymbol{z}, \boldsymbol{\xi})\right| T(\boldsymbol{x}) \mathrm{d} \boldsymbol{x} \mathrm{d} \boldsymbol{\xi} .
\end{aligned}
$$

Here, $A_{p q}(\boldsymbol{x}, \boldsymbol{\xi})=A_{p q}(\boldsymbol{x}, s(\boldsymbol{\xi}), \omega(\boldsymbol{\xi}))$ using the inverse of the map (23) and $B_{p q}(\boldsymbol{z}, \boldsymbol{\xi})$ is similarly defined. $J_{p q}$ is the Jacobian of the inverse of the map (23). Under the assumptions that $A_{p q}$ and $B_{p q}$ satisfy estimates of the form (3), we have that $\mathcal{K}_{p q} \mathcal{F}_{p q}$ is a pseudodifferential operator and, hence, visible edges are reconstructed at the right location. To reconstruct these edges at the right strength, one chooses the filter $B_{p q}$ in such a way that the point spread function (PSF) of $\hat{T}_{p q}(\boldsymbol{z})$ is approximately a Dirac delta function. The choice of $B_{p q}$ for which this is possible is (see [33])

$$
B_{p q}(z, s, \omega)=\frac{\chi_{\Omega_{Z}^{p q}}(s, \omega) \overline{A_{p q}(z, s, \omega)}}{\left|A_{p q}(z, s, \omega)\right|^{2}\left|J_{p q}(z, z, s, \omega)\right|}
$$

where $\chi_{\Omega_{z}^{p q}}(s, \omega)$ is a smooth cut-off function identically 1 in the interior of the set $\Omega_{z}^{p q}$ and 0 outside. The set

$$
\Omega_{z}^{p q}=\left\{\frac{\omega}{c_{0}} \Sigma_{p q}(z, z, s): A_{p q}(z, s, \omega) \neq 0\right\} .
$$

is called the data-collection manifold of the $(p, q)$ transmitterreceiver pair.

In terms of the change of variables (23), we can also describe this data collection manifold as

$$
\Omega_{\boldsymbol{z}}^{p q}=\left\{\frac{\omega(\boldsymbol{\xi})}{c_{0}} \Sigma_{p q}(z, z, s(\boldsymbol{\xi})): A_{p q}(\boldsymbol{z}, \boldsymbol{\xi}) \neq 0\right\} .
$$

Thus, for each $(p, q)$ transmitter-receiver pair, the FBP method of [33] gives an approximate reconstruction of $T$ on the data collection manifold determined by the $p$ th and $q$ th antenna trajectories. The superposition of all the reconstructed images using $d_{p q}, p=1, \ldots, M$ and $q=1, \ldots, N$ results in the final approximate reconstruction of $T$ as described in [6].

\section{Multistatic Image Formation}

While our basic strategy is to employ an FBP method as in the bistatic SAR case - that is by fixing a transmitter-receiver pair, reconstructing $T$, and then superposing all the reconstructed images - multistatic image reconstruction has additional complications and, hence, significant differences. Since we do not assume decomposability of the multistatic received signal $d_{q}$ in (5) into bistatic received signals $d_{p q}$, the bistatic FBP method of [33] is not suitable. This is because the signals at the receiver $q$ from multiple transmitters and the thermal noise interfere and induce artifacts. The important point then is that the filter we derive for the $(p, q)$ transmitter-receiver pair should not only reconstruct the visible edges at the right strength, but also suppress the artifacts due to other transmitters and the thermal noise at the $q$ th receiver.

We suppress the strength of these artifacts and at the same time reconstruct the visible edges of $T$ corresponding to the $(p, q)$ transmitter-receiver pair by, roughly speaking, designing the filter $B_{p q}$ in a minimum mean-square error sense, such that the frequency content of the target in the bistatic data collection manifold of the $(p, q)$ transmitter-receiver pair (26) is preserved, whereas the frequency content of the artifacts is suppressed. The frequency content of the artifacts is contained in the bistatic data collection manifolds $\Omega_{z}^{r q}$ for the $(r, q)$ transmitter-receiver pair with $r \neq p$. These data collection manifold are defined as in (26) by replacing $p$ by $r$. More precisely

$$
\Omega_{z}^{r q}=\left\{\frac{\omega}{c_{0}} \Sigma_{r q}(z, z, s): A_{r q}(z, s, \omega) \neq 0\right\}
$$

with

$$
\Sigma_{r q}(\boldsymbol{z}, \boldsymbol{x}, s)=\int_{0}^{1} \nabla R_{r q}(s, \boldsymbol{z}+\lambda(\boldsymbol{x}-\boldsymbol{z})) \mathrm{d} \lambda
$$

\section{A. Backprojection Operators}

We first define the set of filtered-backprojection operators, $\mathcal{K}_{p q}$, for the multistatic data at the $q$ th receiver with respect to each transmitter $p, p=1, \ldots, M$ as follows:

$$
\mathcal{K}_{p q} d_{q}(\boldsymbol{z}):=\int e^{-\mathrm{i} \omega / c_{0} R_{p q}(s, z)} B_{p q}(z, s, \omega) D_{q}(s, \omega) \mathrm{d} s \mathrm{~d} \omega
$$

where

$$
D_{q}(s, \omega)=\sum_{p=1}^{M} \int e^{\mathrm{i} \omega / c_{0} R_{p q}(s, \boldsymbol{x})} A_{p q}(\boldsymbol{x}, s, \omega) T(\boldsymbol{x}) \mathrm{d} x .
$$

Here $B_{p q}$ for $q=1, \ldots, N$ and $p=1, \ldots, M$ are the filters to be determined.

Let $\hat{T}_{p q}(\boldsymbol{z})$ denote the image reconstructed by $\mathcal{K}_{p q}$, that is

$$
\hat{T}_{p q}(z):=\mathcal{K}_{p q} d_{q}(z) .
$$

In order to study $\hat{T}_{p q}(\boldsymbol{z})$, we decompose the forward model $\mathcal{F}_{q}$ as

$$
\mathcal{F}_{q}=\mathcal{F}_{p q}+\sum_{r=1, r \neq p}^{M} \mathcal{F}_{r q}
$$

Recall that $\mathcal{F}_{q}$ and $\mathcal{F}_{p q}$ are defined in (16) and (1) respectively and $\mathcal{F}_{r q}$ is obtained by replacing $r$ instead of $p$ in (1).

Using this decomposition and substituting the multistatic data model given in (16), we obtain

$$
\hat{T}_{p q}(\boldsymbol{z})=\mathcal{K}_{p q} \mathcal{F}_{p q}[T](z)+\sum_{r=1, r \neq p}^{M} \mathcal{K}_{p q} \mathcal{F}_{r q}[T](z)+\mathcal{K}_{p q}\left[n_{q}\right](z) .
$$

For simplicity we write (34) as

$$
\hat{T}_{p q}(z)=\hat{T}_{p q}^{p}(\boldsymbol{z})+\sum_{r=1, r \neq p}^{M} \hat{T}_{p q}^{r}(\boldsymbol{z})+\mathcal{K}_{p q}\left[n_{q}\right](\boldsymbol{z})
$$


where $\hat{T}_{p q}^{p}(\boldsymbol{z})=\mathcal{K}_{p q} \mathcal{F}_{p q}[T](z)$ and $\hat{T}_{p q}^{r}(z)=\mathcal{K}_{p q} \mathcal{F}_{r q}[T](z)$, for $r=1, \ldots, M, r \neq p$. Note that $\mathcal{K}_{p q} \mathcal{F}_{p q}$ is exactly the imaging operator that appears in the bistatic image reconstruction in (18) summarizing the design of the filter in [33]. In Section III, we showed that this is a pseudodifferential operator and since pseudodifferential operators have pseudolocal property, they put the edges of the target $T$ at the right location and right orientation in $\hat{T}_{p q}(z)$. Now $\mathcal{K}_{p q} \mathcal{F}_{r q}[T]$ for $p \neq r=1, \ldots, M$ involves backprojection of the ideal received signal at the $q$ th receiver due to the $r$ th transmitter for $r \neq p$. The operators $\mathcal{K}_{p q} \mathcal{F}_{r q}$ for $r \neq p$ are in general not pseudodifferential operators, and, hence, the edges of $T$ are not reconstructed at the right location and orientation.

\section{B. Design of the Backprojection Filters}

In this subsection, we employ a minimum mean-square error criterion to determine the filters $B_{p q}$. More precisely, our choice of filter $B_{p q}$ is such that the mean-square error between the image reconstructed by $\mathcal{K}_{p q}$ (32) and the best possible image that could be reconstructed given the trajectories of the transmitter-receiver pair $(p, q)$ is minimized.

The bistatic data collection manifold (27) determines the best possible image that could be reconstructed by the pair $(p, q)$, which is given by [33]

$$
T_{\Omega_{\boldsymbol{Z}}^{p q}}(\boldsymbol{z})=\mathcal{I}_{\Omega_{\boldsymbol{Z}}^{p q}} T(\boldsymbol{z})=\int_{\Omega_{\boldsymbol{Z}}^{p q}} \mathrm{e}^{\mathrm{i}(\boldsymbol{x}-\boldsymbol{z}) \cdot \boldsymbol{\xi}} T(\boldsymbol{x}) \mathrm{d} \boldsymbol{x} \mathrm{d} \boldsymbol{\xi}
$$

Now our choice of filter $B_{p q}$ is such that the mean squareerror

$$
I\left(B_{p q}\right)=\mathrm{E}\left[\int\left|\hat{T}_{p q}(\boldsymbol{z})-T_{\Omega_{z}^{p q}}(\boldsymbol{z})\right|^{2} \mathrm{~d} z\right] \text { is minimized }
$$

where, as before, $\mathrm{E}$ is the expectation operator.

In the rest of this section, we simplify the integrals appearing in (37) using the method of stationary phase and then use these simplified integral expressions to take a variational derivative of $I\left(B_{p q}\right)$. This leads to the determination of the filter $B_{p q}$.

Recalling the decomposition

$$
\hat{T}_{p q}(z)=\hat{T}_{p q}^{p}(z)+\sum_{r=1, r \neq p}^{M} \hat{T}_{p q}^{r}(z)+\mathcal{K}_{p q}\left[n_{q}\right](z)
$$

let us expand the right hand side of (37) to obtain

$$
\begin{aligned}
& I\left(B_{p q}\right)=\mathrm{E}\left[\int\left|\hat{T}_{p q}^{p}(\boldsymbol{z})-T_{\Omega_{z}^{p q}}(\boldsymbol{z})\right|^{2} \mathrm{~d} \boldsymbol{z}\right] \\
& +\mathrm{E}\left[\int\left|\sum_{r=1, r \neq p}^{M} \hat{T}_{p q}^{r}(\boldsymbol{z})\right|^{2} \mathrm{~d} \boldsymbol{z}\right] \\
& +2 \mathrm{E}\left[\sum_{r=1, r \neq p}^{M} \operatorname{Re} \int\left(\hat{T}_{p q}^{p}(\boldsymbol{z})-T_{\Omega_{z}^{p q}}(\boldsymbol{z})\right) \overline{\hat{T}_{p q}^{r}} \mathrm{~d} z\right] \\
& +\mathrm{E}\left[\int\left|\mathcal{K} n_{q}(\boldsymbol{z})\right|^{2} \mathrm{~d} \boldsymbol{z}\right] .
\end{aligned}
$$

For simplicity, we denote the terms on the right hand side of (39) as $I_{1}+I_{2}+I_{3}+I_{4}$.

In order to carry out the stationary phase calculations for these integrals, we use the change of variables (23) and transform the first two terms on the right hand side of (38). We obtain

$$
\begin{aligned}
\hat{T}_{p q}^{p}(\boldsymbol{z})=\int \mathrm{e}^{\mathrm{i}(\boldsymbol{x}-\boldsymbol{z}) \cdot \boldsymbol{\xi}} & B_{p q}(\boldsymbol{z}, \boldsymbol{\xi}) \\
& \times A_{p q}(\boldsymbol{z}, \boldsymbol{\xi})\left|J_{p q}(\boldsymbol{x}, \boldsymbol{z}, \boldsymbol{\xi})\right| T(\boldsymbol{x}) \mathrm{d} \boldsymbol{x} \mathrm{d} \boldsymbol{\xi}
\end{aligned}
$$

where recall $J_{p q}$ is the Jacobian of the inverse of the transformation (23) and

$$
\begin{aligned}
\hat{T}_{p q}^{r}(\boldsymbol{z})=\int e^{\mathrm{i}(\boldsymbol{x}-z) \cdot \boldsymbol{\xi}} B_{p q}(\boldsymbol{z}, \boldsymbol{\xi}) A_{r q}(\boldsymbol{x}, \boldsymbol{\xi}) \\
\times\left|J_{p q}(\boldsymbol{x}, z, \boldsymbol{\xi})\right| \tilde{T}_{r}(\boldsymbol{x}, \boldsymbol{\xi}) \mathrm{d} \boldsymbol{x} \mathrm{d} \boldsymbol{\xi}
\end{aligned}
$$

where

$$
\tilde{T}_{r}(\boldsymbol{x}, \boldsymbol{\xi}):=\tilde{T}_{r}(\boldsymbol{x}, s(\boldsymbol{\xi}), \omega(\boldsymbol{\xi}))=e^{\mathrm{i} \triangle_{r p}(\boldsymbol{x}, s(\boldsymbol{\xi}), \omega(\boldsymbol{\xi}))} T(\boldsymbol{x})
$$

and

$$
\begin{aligned}
& \triangle_{r p}(\boldsymbol{x}, s(\boldsymbol{\xi}), \omega(\boldsymbol{\xi})) \\
& \quad=\frac{\omega(\boldsymbol{\xi})}{c_{0}}\left(\left|\mathbf{x}-\boldsymbol{\gamma}_{T_{r}}(s(\boldsymbol{\xi}))\right|-\left|\mathbf{x}-\boldsymbol{\gamma}_{T_{p}}(s(\boldsymbol{\xi}))\right|\right) .
\end{aligned}
$$

Based on our assumption that $n_{q}$ and $T$ are statistically uncorrelated, the remaining terms in the above expansion

$$
2 \mathrm{E}\left[\operatorname{Re} \int\left(\hat{T}_{p q}(\boldsymbol{z})-T_{\Omega_{z}^{p q}}(\boldsymbol{z})\right) \overline{\mathcal{K}_{p q}\left[n_{q}\right](\boldsymbol{z})} \mathrm{d} z\right]=0
$$

and

$$
2 \mathrm{E}\left[\operatorname{Re} \int \sum_{r=1, r \neq p}^{M} \hat{T}_{p q}^{r}(z) \overline{\mathcal{K}_{p q}\left[n_{q}\right](z)} \mathrm{d} z\right]=0 .
$$

We now proceed to calculate each of the integrals $I_{i}$ for $i=$ $1, \ldots, 4$.

1) Calculation of $I_{1}$ : We have

$$
I_{1}=\mathrm{E}\left[\int\left|\hat{T}_{p q}^{p}(z)-T_{\Omega_{z}^{p q}}(z)\right|^{2} \mathrm{~d} z\right] .
$$

We show in Appendix B that $I_{1}$ has the following integral representation:

$$
I_{1} \approx \int\left|B_{p q}\left(\boldsymbol{x}^{\prime}, \boldsymbol{\zeta}\right) A_{p q}\left(\boldsymbol{x}^{\prime}, \boldsymbol{\zeta}\right)\right| J_{p q}\left(\boldsymbol{x}^{\prime}, \boldsymbol{x}^{\prime}, \boldsymbol{\zeta}\right)|-1|^{2} S_{T}(\boldsymbol{\zeta}) \mathrm{d} x^{\prime} \mathrm{d} \boldsymbol{\zeta}
$$

2) Calculation of $I_{2}$ : We now consider

$$
I_{2}=\mathrm{E}\left[\int\left|\sum_{r=1, r \neq p}^{M} \hat{T}_{p q}^{r}\right|^{2} \mathrm{~d} z\right] .
$$


We show in Appendix $\mathrm{C}$ that $I_{2}$ has the following integral expression:

$$
\begin{array}{r}
I_{2} \approx \sum_{r=1, r \neq p}^{M} \int\left|A_{r q}\left(\boldsymbol{x}^{\prime}, \boldsymbol{\zeta}\right) B_{p q}\left(\boldsymbol{x}^{\prime}, \boldsymbol{\zeta}\right) J_{p q}\left(\boldsymbol{x}^{\prime}, \boldsymbol{x}^{\prime}, \boldsymbol{\zeta}\right)\right|^{2} \\
\times S_{T}\left(\boldsymbol{\zeta}+\partial_{\boldsymbol{x}^{\prime}} \triangle_{r p}(0, \boldsymbol{\zeta})\right) \mathrm{d} \boldsymbol{x}^{\prime} \mathrm{d} \boldsymbol{\zeta} .
\end{array}
$$

3) Calculation of $I_{3}$ : We show in Appendix D that the assumption of stationarity of $S_{T}$ implies that the leading order contribution of $I_{3}$ to $I\left(B_{p q}\right)$ is 0 . Therefore, this integral does not contribute to the determination of the filter $B_{p q}$.

4) Calculation of $I_{4}$ : We have

$$
\begin{aligned}
I_{4}= & \mathrm{E}\left[\int\left|\mathcal{K}\left[n_{q}\right](\boldsymbol{z})\right|^{2} \mathrm{~d} \boldsymbol{z}\right] \\
= & \int e^{\mathrm{i}\left(\omega^{\prime} / c_{0} R_{p q}\left(s^{\prime}, \boldsymbol{z}\right)-\omega / c_{0} R_{p q}(s, \boldsymbol{z})\right)} B_{p q}(\boldsymbol{z}, s, \omega) \\
& \times \overline{B_{p q}\left(\boldsymbol{z}, s^{\prime}, \omega^{\prime}\right)} \widetilde{S}_{n_{q}}\left(s, \omega, s^{\prime}, \omega^{\prime}\right) \mathrm{d} s \mathrm{~d} s^{\prime} \mathrm{d} \omega \mathrm{d} \omega^{\prime} \mathrm{d} \boldsymbol{z} .
\end{aligned}
$$

With condition (14), we obtain

$$
I_{4}=\int\left|B_{p q}(z, s, \omega)\right|^{2} S_{n_{q}}(\omega, s) \mathrm{d} \omega \mathrm{d} s \mathrm{~d} z .
$$

Making the change of variables (23) in this integral, we have

$$
I_{4}=\int\left|B_{p q}(\boldsymbol{z}, \boldsymbol{\zeta})\right|^{2} S_{n}(\boldsymbol{\zeta})|J(\boldsymbol{z}, \boldsymbol{z}, \boldsymbol{\zeta})| \mathrm{d} \boldsymbol{z} \mathrm{d} \boldsymbol{\zeta} .
$$

5) Variational Derivative: We obtain the following contributions to $I\left(B_{p q}\right)$ :

$$
I\left(B_{p q}\right) \approx I_{1}+I_{2}+I_{4}
$$

where $I_{1}, I_{2}$, and $I_{4}$ are given by (47), (49), and (53), respectively. Recall that we wish to choose the filter $B_{p q}$ such that $I\left(B_{p q}\right)$ is a minimum. With the above approximation for $I\left(B_{p q}\right)$, we consider a variational derivative to minimize $I\left(B_{p q}\right)$. That is, we fix a filter $B_{0}$ and consider the variation, $B_{p q}+\varepsilon B_{0}$ for small $\varepsilon$. If $B_{p q}$ is such that $I\left(B_{p q}\right)$ is a minimum, we must have

$$
\left.\frac{\mathrm{d}}{\mathrm{d} \varepsilon}\right|_{\varepsilon=0} I\left(B_{p q}+\varepsilon B_{0}\right)=0
$$

Now this must hold for any $B_{0}$ and so varying $B_{0}$ leads to the determination of the filter $B_{p q}$. The steps are carried out in Appendix E. We obtain

$$
B_{p q}(\boldsymbol{x}, \boldsymbol{\zeta})=\frac{\chi_{\Omega_{x}^{p q}}(\boldsymbol{x}, \boldsymbol{\zeta}) \overline{A_{p q}(\boldsymbol{x}, \boldsymbol{\zeta})} S_{T}(\boldsymbol{\zeta})}{\left|J_{p q}(\boldsymbol{x}, \boldsymbol{x}, \boldsymbol{\zeta})\right| \times \Psi+S_{n_{q}}(\boldsymbol{\zeta})}
$$

where

$$
\begin{aligned}
\Psi=\left|A_{p q}(\boldsymbol{x}, \boldsymbol{\zeta})\right|^{2} S_{T}(\boldsymbol{\zeta}) \\
\quad+\sum_{r=1, r \neq p}^{M}\left|A_{r q}(\boldsymbol{x}, \boldsymbol{\zeta})\right|^{2} S_{T}\left(\boldsymbol{\zeta}+\partial_{\boldsymbol{x}} \triangle_{r q}(0, \boldsymbol{\zeta})\right)
\end{aligned}
$$

Here, $\chi \Omega_{x}^{p q}$ is a smooth cut-off function such that it is identically 1 for those $\boldsymbol{\zeta}$ such that $A_{p q}(\boldsymbol{x}, \boldsymbol{\zeta}) \neq 0$.
We can use (23) to write $B_{p q}$ in terms of $(s, \omega)$. In obtaining (60), it is notationally convenient to make the assumption that the size of the target scene is much smaller compared to the distance of the airborne platforms from the target scene. Note that this assumption is realistic for SAR imaging scenarios. Letting $\boldsymbol{\psi}_{0}=(0,0, \boldsymbol{\psi}(0,0))$, when then have

$$
\begin{aligned}
\partial_{\boldsymbol{x}} \Delta_{r p}(0, \zeta) & =\frac{\omega}{c_{0}}[D(0,0)]^{T}\left(\boldsymbol{\psi}_{0}-\widehat{-\boldsymbol{\gamma}_{T_{r}}}(s)-\boldsymbol{\psi}_{0}-\widehat{\boldsymbol{\gamma}_{T_{p}}}(s)\right) \\
& \approx \frac{\omega}{c_{0}}\left[D\left(x_{1}, x_{2}\right)\right]^{T}\left(\mathbf{x}-\widehat{\boldsymbol{\gamma}_{T_{r}}}(s)-\mathbf{x}-\widehat{\boldsymbol{\gamma}_{T_{p}}}(s)\right)
\end{aligned}
$$

This follows from the assumption above, because for small variations of $\boldsymbol{x}$ from the origin, we have that unit vectors $\mathbf{x}-\widehat{\gamma_{T_{p}}}(s)$ and $\mathbf{x}-\widehat{\boldsymbol{\gamma}_{T_{r}}}(s)$ have small variation in directions from that of $\boldsymbol{\psi}_{0}-\widehat{\boldsymbol{\gamma}_{T_{r}}}(s)$ and $\boldsymbol{\psi}_{0}-\widehat{\boldsymbol{\gamma}_{T_{p}}}(s)$, respectively. Therefore, using (23), we obtain

$\zeta+\partial_{\boldsymbol{x}} \Delta_{r p}(0, \boldsymbol{\zeta}) \approx \frac{\omega}{c_{0}}\left[D\left(x_{1}, x_{2}\right)\right]^{T}\left(\mathbf{x}-\widehat{\gamma_{T_{r}}}(s)+\mathbf{x}-\widehat{\boldsymbol{\gamma}_{R_{q}}}(s)\right)$

Hence, we have

$$
B_{p q}(\boldsymbol{x}, s, \omega) \approx \frac{\chi_{\Omega_{\boldsymbol{x}}^{p q}}(\boldsymbol{x}, s, \omega) \overline{A_{p q}(\boldsymbol{x}, s, \omega)} S_{T}\left(\boldsymbol{\zeta}_{p q}\right)}{\left|J_{p q}(\boldsymbol{x}, \boldsymbol{x}, s, \omega)\right| \times \Psi+S_{n_{q}}\left(\boldsymbol{\zeta}_{p q}\right)}
$$

where

$$
\begin{aligned}
\Psi= & \left|A_{p q}(\boldsymbol{x}, s, \omega)\right|^{2} S_{T}\left(\boldsymbol{\zeta}_{p q}\right) \\
& +\sum_{r=1, r \neq p}^{M}\left|A_{r q}(\boldsymbol{x}, s, \omega)\right|^{2} S_{T}\left(\boldsymbol{\zeta}_{r q}\right) .
\end{aligned}
$$

Here, the vectors $\zeta_{p q}$ and $\zeta_{r q}$ for $r=1, \ldots, M, r \neq p$ are obtained from

$$
\boldsymbol{\zeta}_{m n}=\frac{\omega}{c_{0}}\left[D\left(x_{1}, x_{2}\right)\right]^{T}\left(\mathbf{x}-\widehat{\boldsymbol{\gamma}_{T_{m}}}(s)+\mathbf{x}-\widehat{\gamma_{R_{n}}}(s)\right)
$$

for $m=1, \ldots, M$ and $n=1, \ldots, N$.

\section{Reconstructed Image}

In Sections IV-A and IV-B, we defined the filtered-backprojection operators for a given transmitter-receiver pair and determined the filters by employing a minimum mean-square error criterion. In this section, we give the equations obtaining the final image at each receiver and across all receivers.

1) Image at the qth Receiver: We consider the images reconstructed from the filtered-backprojection operators defined by the transmitter-receiver pair $(p, q)$ for $p=1, \ldots, M$. The superposition of these images gives the final image at the $q$ th receiver. Defining $\hat{T}_{q}(z)$ to be the image at the $q$ th receiver, we have

$$
\begin{aligned}
\hat{T}_{q}(\boldsymbol{z}) & :=\sum_{p=1}^{M} \hat{T}_{p q}(\boldsymbol{z}) \\
& =\sum_{p=1}^{M} \int e^{-\mathrm{i} \omega / c_{0}} B_{p q}(\boldsymbol{z}, s, \omega) D_{q}(s, \omega) d s d \omega
\end{aligned}
$$

where $B_{p q}$ is defined in (58) and (59) and $D_{q}(s, \omega)$ is defined in (31). 


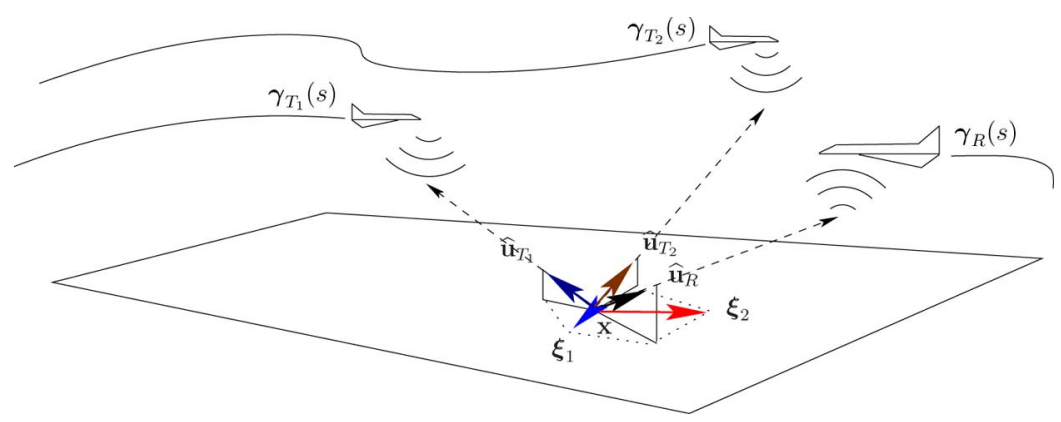

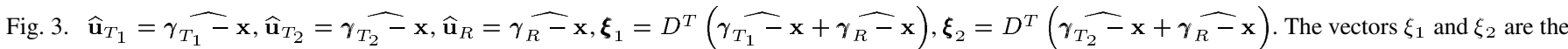
projections on to the ground topography of the vectors $\widehat{\gamma_{T_{1}}} \widehat{-} \mathbf{x}+\widehat{\gamma_{R}-} \mathbf{x}$ and $\widehat{\gamma_{T_{2}}} \widehat{-} \mathbf{x}+\boldsymbol{\gamma}_{R} \widehat{-} \mathbf{x}$, respectively. While backprojecting the received data at the receiver with respect to Transmitter $T_{1}$, the frequency content along direction $\xi_{1}$ of the edge at $\mathbf{x}$ contributes to the image whereas the frequency content along direction $\xi_{2}$ induces the artifact. The reverse argument holds while backprojecting the received data at the receiver with respect to Transmitter $T_{2}$.

2) Image Across all Receivers: To obtain the final image, that is the image across all receivers, we superpose the images obtained at each receiver. Let $\hat{T}(z)$ be the final image. Thus

$$
\begin{aligned}
\hat{T}(z):= & \sum_{q=1}^{N} \hat{T}_{q}(z)=\sum_{q=1}^{N} \sum_{p=1}^{N} \hat{T}_{p q}(z) \\
= & \sum_{q=1}^{N} \sum_{p=1}^{N} \int e^{-\mathrm{i} \omega / c_{0}} B_{p q}(z, s, \omega) \\
& \times D_{q}(s, \omega) \mathrm{d} s \mathrm{~d} \omega .
\end{aligned}
$$

\section{Filters in Special Cases}

To gain a better understanding of the filter $B_{p q}$ that we have derived, let us consider a multistatic SAR geometry with two transmitters and a receiver as shown in Fig. 3. According to our conventions, the transmitter index $p=1,2$, the receiver index $q=1$ and the two filters are denoted by $B_{11}$ and $B_{21}$ and are given by

$$
\begin{aligned}
B_{11}(\boldsymbol{x}, \boldsymbol{\zeta})= & \frac{\chi_{\Omega_{x}^{11}}(\boldsymbol{x}, \boldsymbol{\zeta}) \overline{A_{11}(\boldsymbol{x}, \boldsymbol{\zeta})} S_{T}(\boldsymbol{\zeta})}{\left|J_{11}(\boldsymbol{x}, \boldsymbol{x}, \boldsymbol{\zeta})\right| \times \Psi_{1}+S_{n_{q}}(\boldsymbol{\zeta})} \\
\Psi_{1}= & \left|A_{11}(\boldsymbol{x}, \boldsymbol{\zeta})\right|^{2} S_{T}(\boldsymbol{\zeta}) \\
& +\left|A_{21}(\boldsymbol{x}, \boldsymbol{\zeta})\right|^{2} S_{T}\left(\boldsymbol{\zeta}+\partial_{\boldsymbol{x}} \triangle_{21}(0, \boldsymbol{\zeta})\right)
\end{aligned}
$$

and

$$
B_{21}(\boldsymbol{x}, \boldsymbol{\zeta})=\frac{\chi_{\Omega_{x}^{21}}(\boldsymbol{x}, \boldsymbol{\zeta}) \overline{A_{21}(\boldsymbol{x}, \boldsymbol{\zeta})} S_{T}(\boldsymbol{\zeta})}{\left|J_{21}(\boldsymbol{x}, \boldsymbol{x}, \boldsymbol{\zeta})\right| \times \Psi_{2}+S_{n_{q}}(\boldsymbol{\zeta})}
$$

where

$$
\Psi_{2}=\left|A_{21}(\boldsymbol{x}, \boldsymbol{\zeta})\right|^{2} S_{T}(\boldsymbol{\zeta})+\left|A_{11}(\boldsymbol{x}, \boldsymbol{\zeta})\right|^{2} S_{T}\left(\boldsymbol{\zeta}+\partial_{\boldsymbol{x}} \triangle_{11}(0, \boldsymbol{\zeta})\right) .
$$

When we consider the transmitter-receiver pair $(1,1)$, the power spectral density function $S_{T}$ of the target reflectivity function $T$ along the direction $-\xi_{1}$ in Fig. 3 induces the artifact. In the filter $B_{11}$, the direction $\boldsymbol{\zeta}+\partial_{\boldsymbol{x}} \triangle_{21}(0, \boldsymbol{\zeta})$ (for a particular choice of $(s, \omega)$ ) approximates the direction $-\xi_{1}$ and $S_{T}\left(\boldsymbol{\zeta}+\partial_{\boldsymbol{x}} \triangle_{21}(0, \boldsymbol{\zeta})\right)$ is an approximation to the power spectral density function $S_{T}\left(-\xi_{1}\right)$. The filter $B_{11}$ suppresses the strength of this artifact.

For the filter $B_{21}$ the reverse argument holds. For the transmitter-receiver pair $(2,1), S_{T}$ along $-\xi_{2}$ induces the artifact, and as before for a particular choice of $(s, \omega)$, the direction $\boldsymbol{\zeta}+\partial_{\boldsymbol{x}} \triangle_{11}(0, \boldsymbol{\zeta})$ is an approximation to the direction $-\xi_{2} . B_{21}$ suppresses the strength of this artifact.

We consider the following special cases of the filter $B_{p q}$ :

1) No Additive Thermal Noise: When there is no additive thermal noise at the receivers, we have for each $q=1, \ldots, N$, $S_{n_{q}}=0$ and the filter $B_{p q}$ becomes

$$
B_{p q}(\boldsymbol{x}, \boldsymbol{\zeta})=\frac{\chi_{\Omega_{x}^{p q}}(\boldsymbol{x}, \boldsymbol{\zeta}) \overline{A_{p q}(\boldsymbol{x}, \boldsymbol{\zeta})} S_{T}(\boldsymbol{\zeta})}{\left|J_{p q}(\boldsymbol{x}, \boldsymbol{x}, \boldsymbol{\zeta})\right| \times \Psi}
$$

where $\Psi$ is as in (57).

2) Co-Located Single Transmitter and Single Receiver: If we assume that there is only one transmitter and one receiver and these are co-located then we have a monostatic imaging scenario. Assuming there is no thermal noise at the receiver, our filter becomes

$$
B(\boldsymbol{x}, \boldsymbol{\zeta})=\frac{\chi_{\Omega_{x}}(\boldsymbol{x}, \boldsymbol{\zeta}) \overline{A(\boldsymbol{x}, \boldsymbol{\zeta})}}{|J(\boldsymbol{x}, \boldsymbol{x}, \boldsymbol{\zeta})||A(\boldsymbol{x}, \boldsymbol{\zeta})|^{2}} .
$$

If we assume there is thermal noise at the receiver, our filter becomes

$$
B(\boldsymbol{x}, \boldsymbol{\zeta})=\frac{\chi_{\Omega_{x}}(\boldsymbol{x}, \boldsymbol{\zeta}) \overline{A(\boldsymbol{x}, \boldsymbol{\zeta})} S_{T}(\boldsymbol{\zeta})}{|J(\boldsymbol{x}, \boldsymbol{x}, \boldsymbol{\zeta})||A(\boldsymbol{x}, \boldsymbol{\zeta})|^{2} \mid S_{T}(\boldsymbol{\zeta})+S_{n}(\boldsymbol{\zeta})} .
$$

Since there is only one transmitter and receiver, we have avoided the subscripts $p, q$ in the filters given above. The filters (70) and (71) are the same as the ones obtained in [34].

3) Single Transmitter: Let us assume that there is only one transmitter $p$ in our multistatic setup. Additionally, if one assumes that there is no additive thermal noise at the receivers, the filters we obtain are

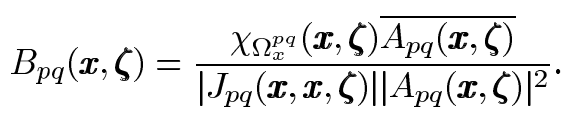


Note that this filter is same as the one derived in [33]. If one assumes that there is additive thermal noise at the receivers, then the filters we have derived become

$$
B_{p q}(\boldsymbol{x}, \boldsymbol{\zeta})=\frac{\chi_{\Omega_{x}^{p q}}(\boldsymbol{x}, \boldsymbol{\zeta}) \overline{A_{p q}(\boldsymbol{x}, \boldsymbol{\zeta})} S_{T}(\boldsymbol{\zeta})}{\left|J_{p q}(\boldsymbol{x}, \boldsymbol{x}, \boldsymbol{\zeta})\right|\left|A_{p q}(\boldsymbol{x}, \boldsymbol{\zeta})\right|^{2} S_{T}(\boldsymbol{\zeta})+S_{n_{q}}(\boldsymbol{\zeta})}
$$

For the case of a single transmitter and single receiver, that is, when $p=q=1$, this filter was derived in [27].

\section{Multistatic IMAGe Formation FOR ANISOTROPIC SCATTERERS}

In the previous sections, we assumed that the scene to be imaged is composed of isotropic scatterers. This assumption simplifies the derivation of our image formation algorithm and distills the important aspects that can be extended.

The assumption of isotropic scatterers is valid for sufficiently small aperture angles [12], [18]. Multistatic synthetic aperture imaging systems are capable of forming relatively wide aperture angles where the isotropic scatterer assumption may not be valid. In this section, we present an extension of our multistatic image formation algorithm to the case involving anisotropic scatterers. Our extension follows the approach outlined in [18]. For each transmitter-receiver pair $(p, q)$, we model the scene reflectivity as a stationary random field $T_{p q}$ with power spectral density function $S_{p q}$. Thus, the received signal model at the $q$ th receiver given in (4) is modified to

$$
\begin{aligned}
d_{q}(s, t)=\sum_{p=1}^{M} \int e^{-\mathrm{i} \omega\left(t-1 / c_{0} R_{p q}(s, \boldsymbol{x})\right)} & \\
& \times A_{p q}(\boldsymbol{x}, s, \omega) T_{p q}(\boldsymbol{x}) \mathrm{d} \boldsymbol{x} \mathrm{d} \omega .
\end{aligned}
$$

We fix a point $\boldsymbol{x}=\left(x_{1}, x_{2}\right)$ and choose an $s_{c} \in\left[s_{0}, s_{1}\right]$, the slow-time interval. Next, consider the following vectors:

$$
\phi_{c}=\left[D\left(x_{1}, x_{2}\right)\right]^{T}\left(\mathbf{x}-\widehat{\boldsymbol{\gamma}_{T_{p}}}\left(s_{c}\right)+\mathbf{x}-\widehat{\gamma_{R_{q}}}\left(s_{c}\right)\right)
$$

and choose a small $\triangle>0$ neighborhood of $\phi_{c}$ of the form

$$
\phi_{s}=\left[D\left(x_{1}, x_{2}\right)\right]^{T}\left(\mathbf{x}-\widehat{\gamma_{T_{p}}}(s)+\mathbf{x}-\widehat{\gamma_{R_{q}}}(s)\right)
$$

for $s$ in a small interval of $s_{c}$. That is, $s$ is close enough to $s_{c}$ such that $\left\|\phi_{s}-\phi_{c}\right\|<\triangle$. Assuming we consider only those vectors $\phi_{s}$ in image formation such that $\left\|\phi_{s}-\phi_{c}\right\|<\triangle$, the assumption of isotropic scattering is valid and, hence, our multistatic image reconstruction algorithm applies.

Now the filter that reconstructs the image $\hat{T}_{p q}^{\phi_{c}}, \triangle$ is

$$
B_{p q}^{\phi_{c}, \triangle}(\boldsymbol{x}, s, \omega) \approx \frac{\chi_{\Omega_{x}^{p q, \phi_{c}, \Delta}}(\boldsymbol{x}, s, \omega) \overline{A_{p q}(\boldsymbol{x}, s, \omega)} S_{T_{p q}}\left(\boldsymbol{\zeta}_{p q}\right)}{\left|J_{p q}(\boldsymbol{x}, \boldsymbol{x}, s, \omega)\right| \times \Psi+S_{n_{q}}\left(\boldsymbol{\zeta}_{p q}\right)}
$$

where

$$
\begin{aligned}
\Psi=\left|A_{p q}(\boldsymbol{x}, s, \omega)\right|^{2} & S_{T_{p q}}\left(\boldsymbol{\zeta}_{p q}\right) \\
& +\sum_{r=1, r \neq p}^{M}\left|A_{r q}(\boldsymbol{x}, s, \omega)\right|^{2} S_{T_{r q}}\left(\boldsymbol{\zeta}_{r q}\right) .
\end{aligned}
$$

In (77), $\chi_{\Omega_{\boldsymbol{x}}^{p q, \phi_{c}, \triangle}}(\boldsymbol{x}, s, \omega)$ is a smooth cut-off function that is identically 1 over the subset of $(s, \omega)$ such that $s$ is close enough to $s_{c}$ with $\left\|\phi_{s}-\phi_{c}\right\|<\triangle$ and $A_{p q}(\boldsymbol{x}, s, \omega) \neq 0$ and the vectors $\vec{v}_{p q}$ and $\vec{v}_{r q}$ are obtained from (60). The final image corresponding to the $(p, q)$ transmitter-receiver pair may be formed by an incoherent combination technique. One such combination is given in [18]

$$
\hat{T}_{p q}(\boldsymbol{x})=\max _{\phi_{c}, \triangle} \hat{T}_{p q}^{\phi_{c}, \triangle}(\boldsymbol{x})
$$

The image at the $q$ th receiver and the final image across all receivers can again be obtained by an incoherent combination method such as root mean square averaging or a mozaicing technique [14].

\section{NUMERICAL SIMULATIONS}

We assume that the scene to be imaged is a realization of a zero-mean stationary process. Furthermore we assume that $a$ priori information on the spectral density function of the scene is available. Such a priori information can be obtained from a secondary imaging modality such as optical imaging. We estimate the power spectral density function of the scene by a periodogram estimator, that is, by computing the magnitude of the discrete Fourier transform of the scene.

For the numerical simulations, we considered a square scene of length $22 \mathrm{~km}$ with a square target of length $5.5 \mathrm{~km}$ with center at $(8.8,12)$ and a rectangular target of length $8.8 \mathrm{~km}$ and width $3.3 \mathrm{~km}$ with center located at $(15.4,10)$ as shown in Fig. 4(a). The scene was discretized to a $128 \times 128$ grid of pixels with $(0$, $0)$ and $(22,22)$ corresponding to pixel numbers $(1,1)$ and $(128$, 128) respectively.

Note that since complex scenes can be represented as a linear combination of rectangles, it suffices to evaluate the performance of our algorithm for the scene shown in Fig. 4(a) due to the linearity of our algorithm.

We performed seven sets of numerical simulations, the results of which can be seen in Figs. 6-12. The transmitter and receiver antenna geometry for these simulations are as follows: 1) Two transmitters and one receiver on a circular path, 2) three transmitters and one receiver on a circular path, 3) two transmitters on linear paths and a receiver on a circular path, 4) three transmitters on linear paths and a receiver on a circular path, 5) two stationary transmitters and one receiver on a circular path, 6) three stationary transmitters and one receiver on a circular path, 7) two transmitters and one receiver on a circular path with additive white Gaussian noise added to the received data.

We made the assumptions that the earth's surface is flat and the amplitude functions $A_{p q} \equiv 1$. This choice of amplitude functions corresponds to isotropic transmitters and receivers using a delta function as the transmit waveform. We used a discrete version of the multistatic forward data model [(4) with $A_{p q} \equiv 1$ and (16)] to generate our simulation data. The parameters we used correspond to a system bandwidth of approximately $0.873 \mathrm{MHz}$.

The circular flight trajectory in our simulations was $\boldsymbol{\gamma}_{c}(s)=$ $(11+11 \cos s, 11+11 \sin s, 6.5)$ [see Fig. 4(b)] and the linear trajectories were, one trajectory parallel to the $x$ axis, $\gamma_{l_{1}}(s)=$ $((22 / 2 \pi) s, 0,6.5)$, and two trajectories parallel to the $y$ axis, 


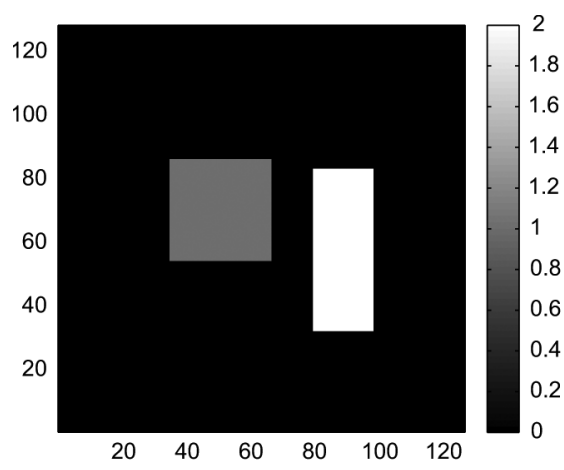

(a)

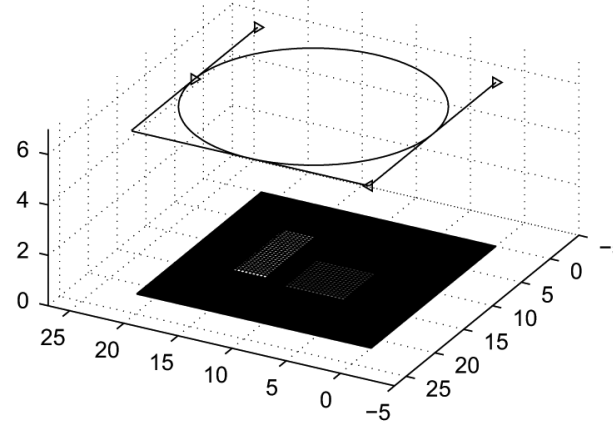

(c)

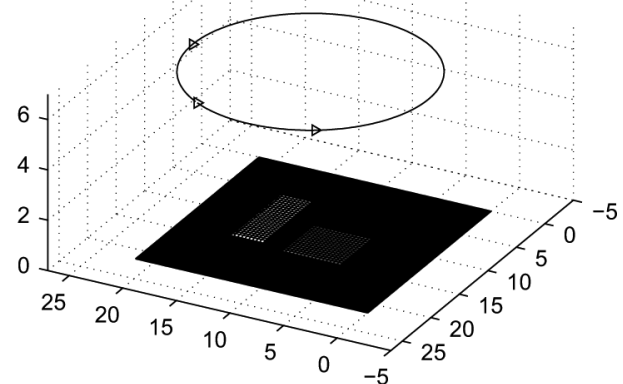

(b)

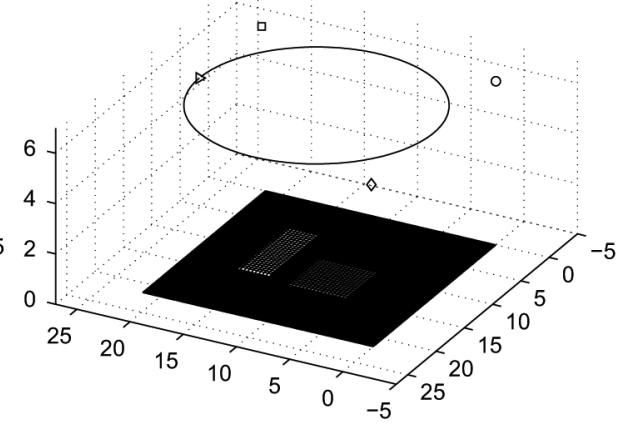

(d)

Fig. 4. (a) Target scene used in numerical simulations. The axes are labeled according to pixel number. (b) 3-D view of the circular trajectory of the transmitters/ receivers used in our simulations. The left and the right arrows are the transmitters and the middle arrow is the receiver, all traversing in the anti-clockwise direction. This is the scenario of Simulations 1 and 7 given by Figs. 5 and 11, respectively. (c) 3-D view of the straight line trajectories of the transmitters and the circular trajectory of the receiver. This imaging scenario is used in Simulation 4 (see Fig. 8). Simulation 3 has a similar scenario with only two transmitters in straight line trajectories above the $x$ axis and $y$ axis (see Fig. 7). (d) 3-D view of the stationary transmitters and the circular trajectory of the receiver. This setup is used in Simulation 6 (see Fig. 10) and in Simulation 5 (see Fig. 9), there are two stationary transmitters and one receiver along the same circular trajectory.

$\gamma_{l_{2}}(s)=(0,(22 / 2 \pi) s, 6.5)$ and $\boldsymbol{\gamma}_{l_{3}}(s)=(22,(22 / 2 \pi) s, 6.5)$ [see Fig. 4(c)]. The stationary transmitters were fixed above the $x$ axis at $(0,0,6.5),(0,22,6.5)$ and above the $y$ axis at $(0,22$, 6.5) [see Fig. 4(d)].

Note that the interference from multiple transmitters results in an artifact in the image. In general the strength of the artifact depends on the power of the transmitters and the geometry of the transmitters and receivers. (Note that in our simulations all transmitters transmit equal power and are roughly of equal distance from the scene to be imaged.) This artifact is structured due to the structured nature of the scene imaged. Therefore, the superposition of reconstructed images using bistatic image formation technique is not expected to cancel out such artifacts.

In our simulations, we compared our multistatic reconstruction scheme with the bistatic reconstruction method of [33]. We see that the reconstruction algorithm introduced in this paper is able to reconstruct the image and at the same time reduce the artifacts induced by using the bistatic reconstruction algorithm of [33]. For the scenario with two transmitters and one receiver on a circular path [Fig. 4(b)], we also considered the case where the received data is corrupted by additive white Gaussian noise.

We define the signal-to-noise ratio (SNR) by

$$
\mathrm{SNR}=20 \log \frac{\frac{1}{N_{d}} \sum_{i=1}^{N_{d}}\left(d\left(s_{i}, t_{i}\right)-\mu_{d}\right)^{2}}{E\left[\|n\|^{2}\right]} \mathrm{dB}
$$

where $N_{T}$ and $N_{d}$ are the number of grid points and and $\mu_{d}$ is the mean value of the radar data.

The mean-square error (MSE) is defined as follows:

$$
\mathrm{MSE}=\frac{\sum_{j=1}^{M} \sum_{i=1}^{N_{T}}\left|T\left(\boldsymbol{x}_{i}\right)-\hat{T}_{j}\left(\boldsymbol{x}_{i}\right)\right|^{2}}{N_{T} M}
$$

where $M$ is the number of reconstructions and $\hat{T}_{j}\left(\boldsymbol{x}_{i}\right)$ is the reconstruction for the $j$ th realization of the noise process.

Fig. 12 shows the MSE curves for a white noise process using both the multistatic filter with noise suppression given in (56) and the filter without noise suppression in (69). As can be seen from the figure the MSE is reduced in cases where there is a low SNR.

\section{CONCLUSION}

In this paper, we developed an FBP-type synthetic-aperture radar inversion method for multistatic SAR involving multiple receivers and multiple transmitters transmitting arbitrary waveforms and multiple receivers. The transmitted waveforms could be overlapping in time and frequency and are not separated by any form of multiplexing. In the design of our FBP-type algorithm, we made the assumption that we have a priori knowledge of the power spectral density function of the target. Such 


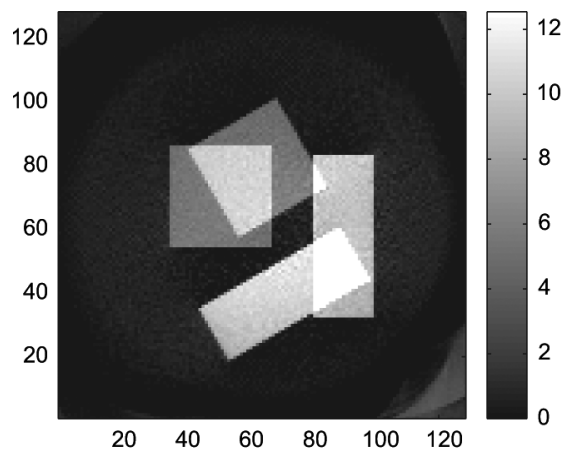

(a)

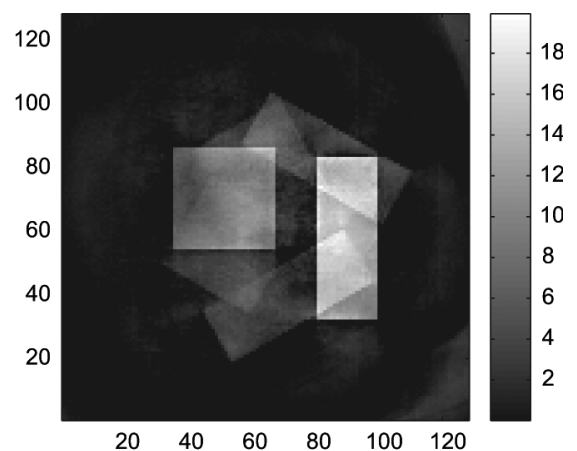

(b)

Fig. 5. Multistatic imaging scenario with two transmitters, $\boldsymbol{\gamma}_{T_{1}}(s)=(11+11 \cos s, 11+11 \sin s, 6.5)$ and $\boldsymbol{\gamma}_{T_{2}}(s)=(11+11 \cos (s+2 \pi / 3), 11+11 \sin (s+$ $2 \pi / 3), 6.5)$, and one receiver, $\gamma_{R}(s)=(11+11 \cos (s+\pi / 3), 11+11 \sin (s+\pi / 3), 6.5)$. (a) Reconstructions from the bistatic FBP algorithm of [33] with respect to Transmitter $T_{2}$. Using the same reconstruction scheme with respect to Transmitter $T_{1}$ introduces additional artifacts [not shown in Fig. 5(a)]. (b) The image reconstructed by multistatic scheme of this paper. Note that the reconstruction method of this paper suppresses both these sets of artifacts.

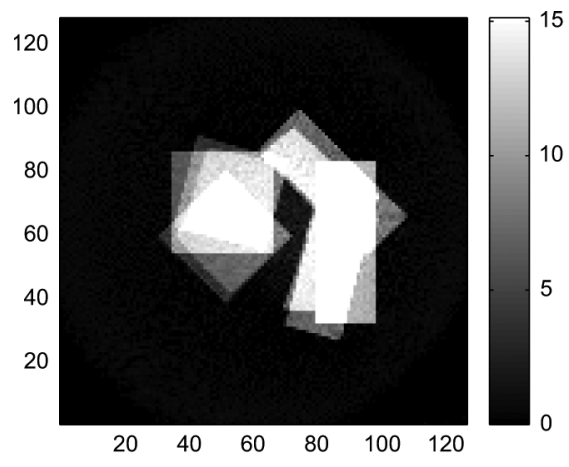

(a)

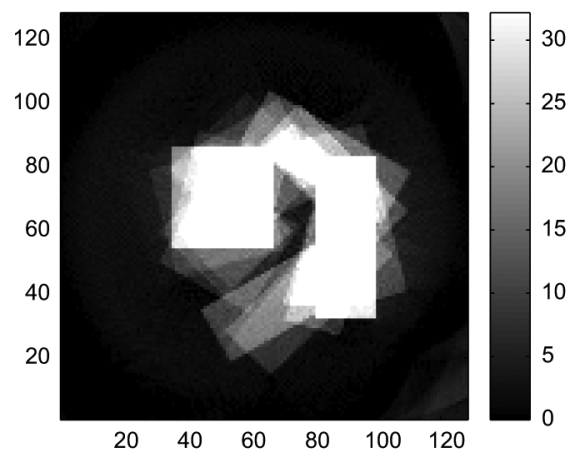

(c)

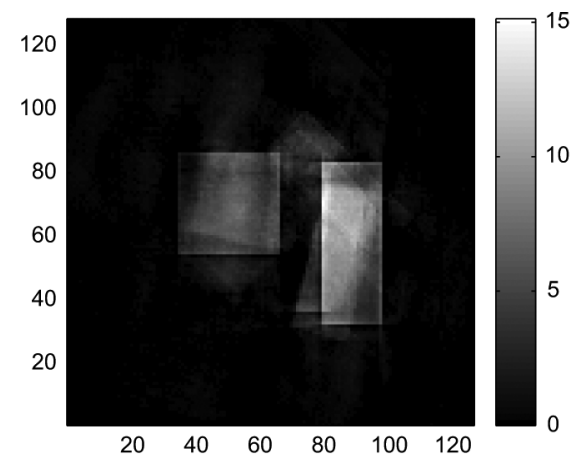

(b)

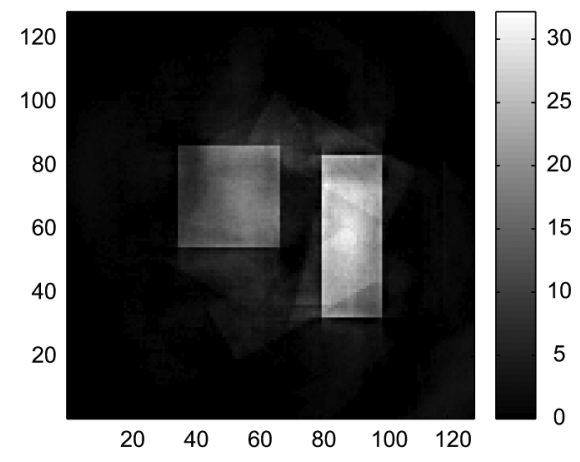

(d)

Fig. 6. Multistatic setup with three transmitters $\boldsymbol{\gamma}_{T_{1}}(s)=(11+11 \cos s, 11+11 \sin s, 6.5), \boldsymbol{\gamma}_{T_{2}}(s)=(11+11 \cos (s+2 \pi / 3), 11+11 \sin (s+2 \pi / 3), 6.5)$ and $\gamma_{T_{3}}(s)=(11+11 \cos (s+\pi / 6), 11+11 \sin (s+\pi / 6), 6.5)$, and one receiver $\gamma_{R}(s)=(11+11 \cos (s+\pi / 3), 11+11 \sin (s+\pi / 3), 6.5)$. (a) Reconstruction from the bistatic FBP algorithm of [33] with respect to Transmitter $T_{3}$. (b) Reconstruction from the multistatic FBP algorithm of this paper with respect to Transmitter $T_{3}$. (c) Superposition of images reconstructed from the bistatic FBP algorithm of [33]. (d) The image reconstructed by the multistatic scheme of this paper. The edges are more visible and the artifacts are greatly reduced due to an additional transmitter involved in imaging the scene and also due to the smaller angle between any transmitter and the receiver.

a priori information can be obtained from a secondary imaging modality. Alternatively, the power spectral density function of the scene can be estimated simultaneously with the image from scattered measurements. However, such an approach is beyond the scope of our current work. We plan to address this problem in the future.

To the best of our knowledge, our work is the first in the literature that can produce multistatic SAR images while suppressing the artifacts caused by multiple interfering transmitters. We demonstrated the performance of the inversion method in numerical simulations, which is in correspondence with theoretical expectations.
While our paper has focused primarily on image formation for multistatic SAR, the techniques are also applicable to other imaging problems such as those arising in acoustics, geophysics and tomography.

Our approach can be exploited to address autofocus problem for multistatic SAR and to suppress artifacts due to multipath effects. These will be the focus of our future work.

\section{APPENDIX A \\ STATIONARY PHASE METHOD}

Let $u$ be a smooth function of compact support in $\mathbb{R}^{n}$ and let $\varphi$ be a real valued function with only nondegenerate critical 


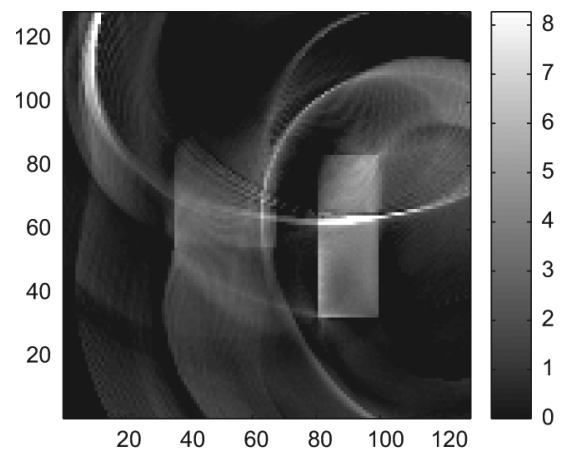

(a)

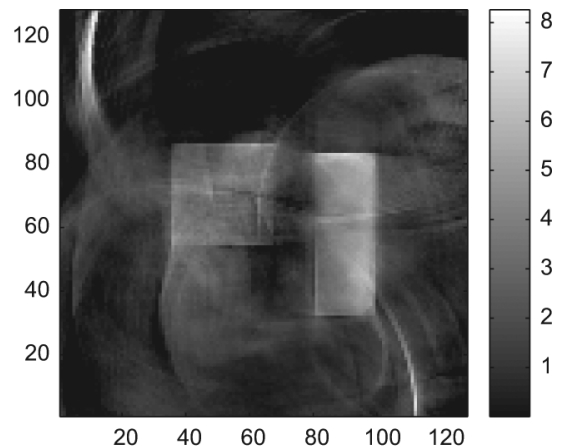

(b)

Fig. 7. Multistatic setup with two transmitters on linear trajectories, $\boldsymbol{\gamma}_{T_{1}}(s)=(0,(22 / 2 \pi) s, 6.5)$ and $\boldsymbol{\gamma}_{T_{2}}(s)=((22 / 2 \pi) s, 22,6.5)$, and one receiver $\gamma_{R}(s)=(11+11 \cos s, 11+11 \sin s, 6.5)$. (a) Reconstructions from the bistatic FBP algorithm of [33] with respect to Transmitter $T_{2}$. (b) The image reconstructed by the multistatic scheme of this paper. Fewer edges of the scene are visible when compared to the previous two images, Figs. 5(b) and 6(d), because the data collection manifold for this geometry has fewer directions when compared to that of the geometry in Figs. 5 and 6 . Therefore, the frequency content of the edges is less leading to some edges being not clearly visible.
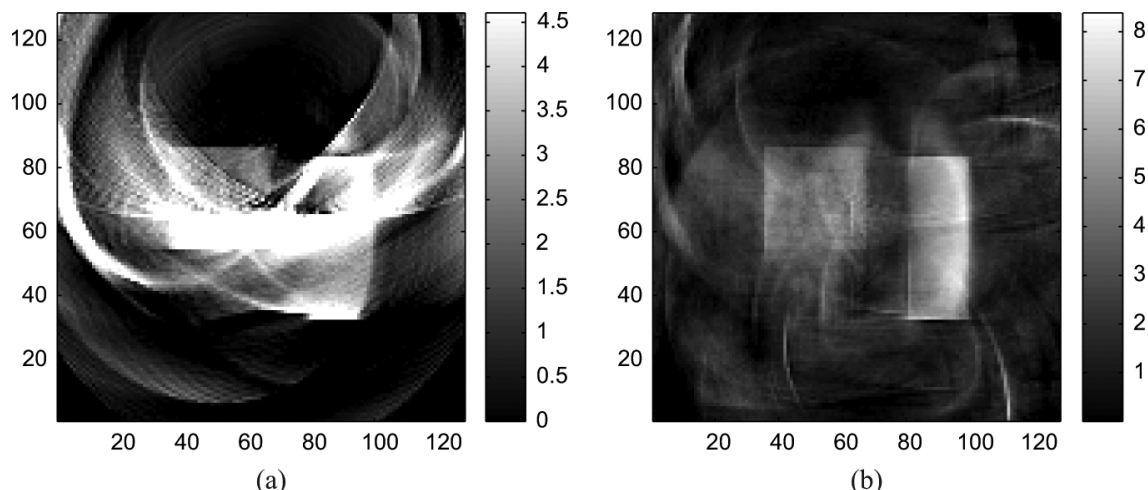

$\begin{array}{llllll}20 & 40 & 60 & 80 & 100 & 120\end{array}$

(b)

Fig. 8. Multistatic setup with three transmitters on linear trajectories, $\gamma_{T_{1}}(s)=(0,22 / 2 \pi, 6.5), \gamma_{T_{2}}(s)=(22 / 2 \pi, 22,6.5)$ and $\gamma_{T_{3}}(s)=$ $(22,22 / 2 \pi, 6.5)$, and one receiver a circular trajectory, $\gamma_{R}(s)=(11+11 \cos s, 11+11 \sin s, 6.5)$. (a) Reconstruction from the bistatic FBP method of [33] with respect to Transmitter $T_{3}$. (b) The image reconstructed by the multistatic algorithm of this paper. While the multistatic geometry is similar to that of Fig. 7 , the addition of one transmitter to the geometry leads to increased frequency content of the edges, and, hence, they are more visible.

points. A point $x_{0} \in \mathbb{R}^{n}$ is called a nondegenerate critical point if $D \varphi\left(x_{0}\right)=0$ and the Hessian matrix $D^{2} \varphi\left(x_{0}\right)$ has nonzero determinant. The stationary phase theorem states that as $\lambda \rightarrow \infty$ [see (82), shown at the bottom of the page].

\section{APPENDIX B}

Stationary Phase Calculation of $I_{1}$

$$
\begin{aligned}
I_{1}= & \mathrm{E}\left[\int\left|\hat{T}_{p}^{p}-T_{\Omega_{z}^{p q}}\right|^{2} \mathrm{~d} \boldsymbol{z}\right] \\
= & \int e^{\mathrm{i}\left((\boldsymbol{x}-\boldsymbol{z}) \cdot \boldsymbol{\xi}-\left(\boldsymbol{x}^{\prime}-\boldsymbol{z}\right) \cdot \boldsymbol{\xi}^{\prime}\right)} \alpha_{p q}(\boldsymbol{x}, \boldsymbol{z}, \boldsymbol{\xi}) \\
& \times \overline{\alpha_{p q}\left(\boldsymbol{x}^{\prime}, \boldsymbol{z}, \boldsymbol{\xi}^{\prime}\right)} R_{T}\left(\boldsymbol{x}, \boldsymbol{x}^{\prime}\right) \mathrm{d} \boldsymbol{x} \mathrm{d} \boldsymbol{x}^{\prime} \mathrm{d} \boldsymbol{\xi} \mathrm{d} \boldsymbol{\xi}^{\prime} \mathrm{d} \boldsymbol{z}
\end{aligned}
$$

where

$$
\alpha_{p q}(\boldsymbol{x}, z, \boldsymbol{\xi})=B_{p q}(\boldsymbol{z}, \boldsymbol{\xi}) A_{p q}(\boldsymbol{x}, \boldsymbol{\xi})\left|J_{p q}(\boldsymbol{x}, \boldsymbol{z}, \boldsymbol{\xi})\right|-1
$$

We apply the method of stationary phase in the variables $z$ and $\boldsymbol{\xi}^{\prime}$. We denote the phase function

$$
\varphi_{1}\left(\boldsymbol{x}, \boldsymbol{x}^{\prime}, z, \boldsymbol{\xi}, \boldsymbol{\xi}^{\prime}\right)=(\boldsymbol{x}-z) \cdot \boldsymbol{\xi}-\left(\boldsymbol{x}^{\prime}-\boldsymbol{z}\right) \cdot \boldsymbol{\xi}^{\prime} .
$$

Differentiating $\varphi_{1}$ with respect to $z$ and $\boldsymbol{\xi}^{\prime}$ and setting it equal to 0 , we have the critical points at

$$
\boldsymbol{\xi}^{\prime}=\boldsymbol{\xi} \text { and } z=\boldsymbol{x}^{\prime}
$$

Now by the method of stationary phase, we obtain

$$
\begin{aligned}
I_{1} \approx \int e^{\mathrm{i}\left(\boldsymbol{x}-\boldsymbol{x}^{\prime}\right) \cdot \boldsymbol{\xi}} \alpha_{p q}\left(\boldsymbol{x}, \boldsymbol{x}^{\prime}, \boldsymbol{\xi}\right) \overline{\alpha_{p q}\left(\boldsymbol{x}^{\prime}, \boldsymbol{x}^{\prime}, \boldsymbol{\xi}\right)} & \\
& \times R_{T}\left(\boldsymbol{x}, \boldsymbol{x}^{\prime}\right) \mathrm{d} \boldsymbol{x} \mathrm{d} \boldsymbol{x}^{\prime} \mathrm{d} \boldsymbol{\xi} .
\end{aligned}
$$

Writing

$$
R_{T}\left(\boldsymbol{x}, \boldsymbol{x}^{\prime}\right)=\int e^{-\mathrm{i}\left(\boldsymbol{x} \cdot \zeta-\boldsymbol{x}^{\prime} \cdot \boldsymbol{\zeta}^{\prime}\right)} \tilde{S}_{T}\left(\boldsymbol{\zeta}, \boldsymbol{\zeta}^{\prime}\right) \mathrm{d} \boldsymbol{\zeta} \mathrm{d} \boldsymbol{\zeta}^{\prime}
$$

$$
\int e^{\mathrm{i} \lambda \varphi(\boldsymbol{x})} u(\boldsymbol{x}) \mathrm{d} \boldsymbol{x}=\left(\frac{2 \pi}{\lambda}\right)^{n / 2} \sum_{\left\{\boldsymbol{x}_{0}: D \varphi\left(\boldsymbol{x}_{0}\right)=0\right\}} \frac{e^{\mathrm{i} \lambda \varphi\left(\boldsymbol{x}_{0}\right)} e^{\mathrm{i} \pi / 4 \operatorname{sgn} D^{2} \varphi\left(\boldsymbol{x}_{0}\right)}}{\sqrt{\left|\operatorname{det} D^{2} \varphi\left(\boldsymbol{x}_{0}\right)\right|}} u\left(x_{0}\right)+\mathcal{O}\left(\lambda^{-n / 2-1}\right)
$$




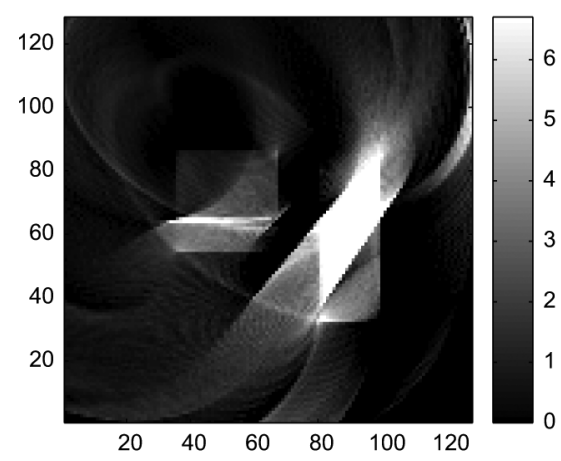

(a)

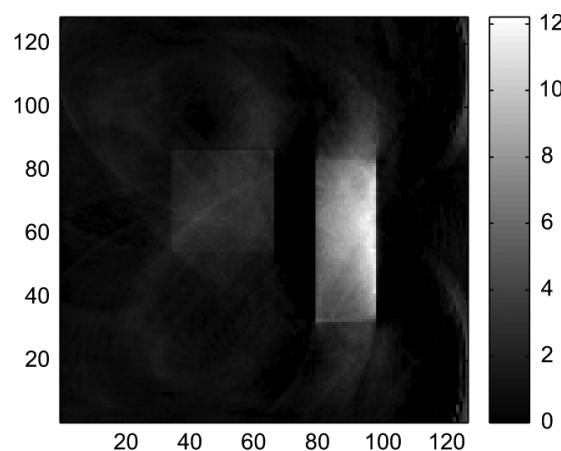

(b)

Fig. 9. Multistatic setup with two stationary transmitters, $\boldsymbol{\gamma}_{T_{1}}(s)=(0,0,6.5)$ and $\boldsymbol{\gamma}_{T_{2}}(s)=(0,22,6.5)$, and one receiver on the circular trajectory, $\boldsymbol{\gamma}_{R}(s)=$ $(11+11 \cos s, 11+11 \sin s, 6.5)$. (a) Reconstruction from the bistatic FBP of [33] with respect to Transmitter $T_{2}$. (b) Reconstruction from the multistatic scheme of this paper. The artifacts appearing in Fig. 9(a) are greatly reduced in Fig. 9(b). The data collection manifolds for this geometry has significantly lesser directions when compared to the previous geometries of Figs. 5-8. This leads to fewer edges being clearly visible.

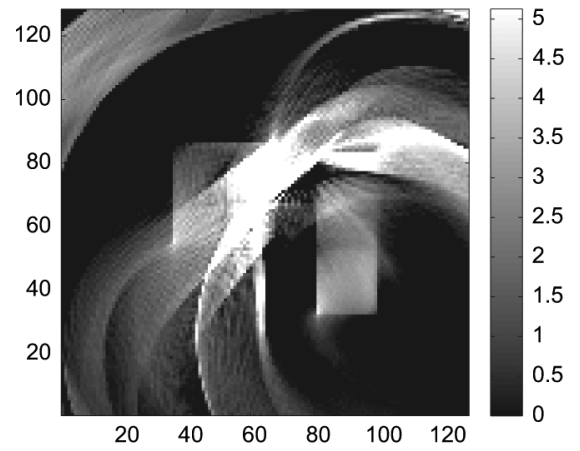

(a)

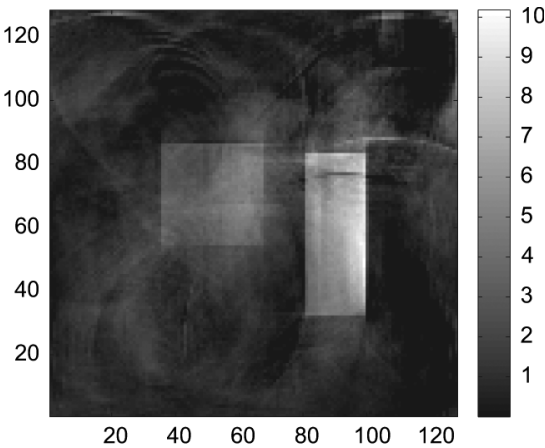

(b)

Fig. 10. Multistatic setup with three stationary transmitters, $\boldsymbol{\gamma}_{T_{1}}(s)=(0,0,6.5), \boldsymbol{\gamma}_{T_{2}}(s)=(22,0,6.5)$ and $\boldsymbol{\gamma}_{T_{3}}(s)=(0,22,6.5)$, and one receiver, $\boldsymbol{\gamma}_{R}(s)=$ $(11+11 \cos s, 11+11 \sin s, 6.5)$. (a) Bistatic FBP reconstruction of [33] with respect to Transmitter $T_{3}$. (b) Reconstruction from the multistatic scheme of this paper. The addition of one more transmitter in imaging the scene, despite it being stationary, does improve the visibility of the reconstructed edges.

we obtain

$$
\begin{aligned}
I_{1} \approx \int e^{\mathrm{i}\left(\left(\boldsymbol{x}-\boldsymbol{x}^{\prime}\right) \cdot \boldsymbol{\xi}-\boldsymbol{x} \cdot \boldsymbol{\zeta}+\boldsymbol{x}^{\prime} \cdot \boldsymbol{\zeta}^{\prime}\right)} \alpha_{p q}\left(\boldsymbol{x}, \boldsymbol{x}^{\prime}, \boldsymbol{\xi}\right) \\
\times \overline{\alpha_{p q}\left(\boldsymbol{x}^{\prime}, \boldsymbol{x}^{\prime}, \boldsymbol{\xi}\right)} \tilde{S}_{T}\left(\boldsymbol{\zeta}, \boldsymbol{\zeta}^{\prime}\right) \mathrm{d} \boldsymbol{x} \mathrm{d} \boldsymbol{x}^{\prime} \mathrm{d} \boldsymbol{\xi} \mathrm{d} \boldsymbol{\zeta} \mathrm{d} \boldsymbol{\zeta}^{\prime} .
\end{aligned}
$$

We now apply the method of stationary phase in the variables, $\boldsymbol{x}$ and $\boldsymbol{\xi}$. We denote the phase function

$$
\varphi_{2}\left(\boldsymbol{x}, \boldsymbol{x}^{\prime}, \boldsymbol{\zeta}, \boldsymbol{\zeta}^{\prime}, \boldsymbol{\xi}\right)=\left(\boldsymbol{x}-\boldsymbol{x}^{\prime}\right) \cdot \boldsymbol{\xi}-\boldsymbol{x} \cdot \boldsymbol{\zeta}+\boldsymbol{x}^{\prime} \cdot \boldsymbol{\zeta}^{\prime} .
$$

Differentiate $\varphi_{2}$ with respect to $\boldsymbol{x}$ and $\boldsymbol{\xi}$ and set it to equal to 0 to obtain the critical points

$$
\boldsymbol{\xi}=\boldsymbol{\zeta} \text { and } \boldsymbol{x}=\boldsymbol{x}^{\prime}
$$

Again by the method of stationary phase, we have

$$
I_{1} \approx \int e^{\mathrm{i} \boldsymbol{x}^{\prime} \cdot\left(\boldsymbol{\zeta}^{\prime}-\boldsymbol{\zeta}\right)}\left|\alpha_{p q}\left(\boldsymbol{x}^{\prime}, \boldsymbol{x}^{\prime}, \boldsymbol{\zeta}\right)\right|^{2} \tilde{S}_{T}\left(\boldsymbol{\zeta}, \boldsymbol{\zeta}^{\prime}\right) \mathrm{d} \boldsymbol{x}^{\prime} \mathrm{d} \boldsymbol{\zeta} \mathrm{d} \boldsymbol{\zeta}^{\prime}
$$

Assuming stationarity of $S_{T}$, we have

$$
I_{1} \approx \int\left|B_{p q}\left(\boldsymbol{x}^{\prime}, \boldsymbol{\zeta}\right) A_{p q}\left(\boldsymbol{x}^{\prime}, \boldsymbol{\zeta}\right)\right| J_{p q}\left(\boldsymbol{x}^{\prime}, \boldsymbol{x}^{\prime}, \boldsymbol{\zeta}\right)|-1|^{2} S_{T}(\boldsymbol{\zeta}) \mathrm{d} x^{\prime} \mathrm{d} \boldsymbol{\zeta}
$$

$$
\begin{gathered}
\text { ApPendix C } \\
\text { Stationary Phase Calculation OF } I_{2} \\
I_{2}=\mathrm{E}\left[\int\left|\sum_{r=1, r \neq p}^{M} \hat{T}_{p q}^{r}\right|^{2} \mathrm{~d} z\right] .
\end{gathered}
$$

Consider

Expanding the right hand side we obtain (94), shown at the bottom of the page, where

$$
\beta_{p q s}(\boldsymbol{x}, \boldsymbol{z}, \boldsymbol{\xi})=B_{p q}(\boldsymbol{z}, \boldsymbol{\xi}) A_{s q}(\boldsymbol{x}, \boldsymbol{\xi})\left|J_{p q}(\boldsymbol{x}, \boldsymbol{z}, \boldsymbol{\xi})\right| .
$$

$$
\begin{aligned}
I_{2}= & \sum_{\substack{r, u=1, r, u \neq p\\
}}^{M} \int e^{\mathrm{i}\left[(\boldsymbol{x}-\boldsymbol{z}) \cdot \boldsymbol{\xi}-\left(\boldsymbol{x}^{\prime}-z\right) \cdot \boldsymbol{\xi}^{\prime}\right]} \beta_{p q r}(\boldsymbol{x}, \boldsymbol{z}, \boldsymbol{\xi}) \overline{\beta_{p q u}\left(\boldsymbol{x}^{\prime}, \boldsymbol{z}, \boldsymbol{\xi}^{\prime}\right)} \\
& \times e^{\mathrm{i}\left(\triangle_{r p}(\boldsymbol{x}, \boldsymbol{\xi})-\triangle_{u p}\left(\boldsymbol{x}^{\prime}, \boldsymbol{\xi}^{\prime}\right)\right)} R_{T}\left(\boldsymbol{x}, \boldsymbol{x}^{\prime}\right) \mathrm{d} \boldsymbol{x} \mathrm{d} \boldsymbol{x}^{\prime} \mathrm{d} \boldsymbol{\xi} \mathrm{d} \boldsymbol{\xi}^{\prime} \mathrm{d} z
\end{aligned}
$$




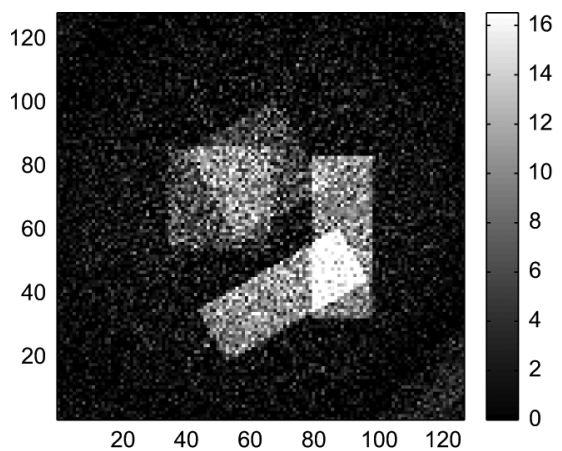

(a)

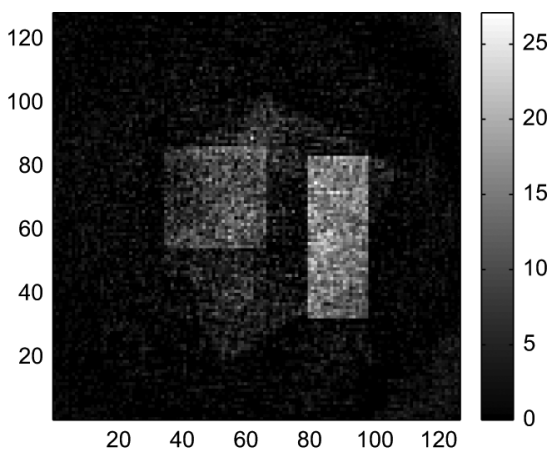

(b)

Fig. 11. Multistatic setup with two transmitters and one receiver with data corrupted with additive white Gaussian noise at an SNR of $20 \mathrm{~dB}$. The flight trajectories are $\gamma_{T_{1}}(s)=(11+11 \cos s, 11+11 \sin s, 6.5), \gamma_{T_{2}}(s)=(11+11 \cos (s+2 \pi / 3), 11+11 \sin (s+2 \pi / 3), 6.5)$, and $\gamma_{R}(s)=(11+11 \cos (s+\pi / 3), 11+$ $11 \sin (s+\pi / 3), 6.5)$. (a) Reconstruction of the target scene using the bistatic FBP scheme of [27] with respect to Transmitter $T_{2}$. (b) Reconstruction from the multistatic algorithm of this paper.

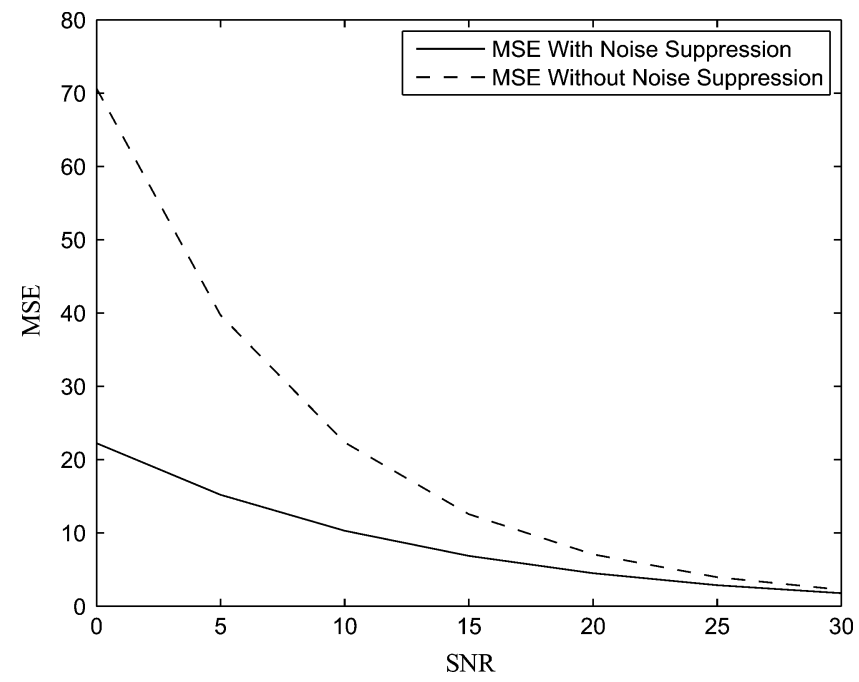

Fig. 12. MSE (vertical axis) versus SNR (horizontal axis) using the multistatic reconstruction algorithm of this paper averaged over ten reconstructions at each SNR level. The simulation setup is the same as in the case of Fig. 11.

Now we obtain a simplified expression for

$$
e^{\mathrm{i}\left(\triangle_{r p}(\boldsymbol{x}, \boldsymbol{\xi})-\triangle_{u p}\left(\boldsymbol{x}^{\prime}, \boldsymbol{\xi}^{\prime}\right)\right)} R_{T}\left(\boldsymbol{x}, \boldsymbol{x}^{\prime}\right) .
$$

To do so we first expand $\triangle_{r p}(\cdot, \boldsymbol{\xi})$ and $\triangle_{u p}\left(\cdot, \boldsymbol{\xi}^{\prime}\right)$ near the origin and letting $\frac{\partial}{\partial \boldsymbol{x}} \triangle_{r p}(0, \boldsymbol{\xi})$ denote $\left.\frac{\partial}{\partial \boldsymbol{x}} \triangle_{r p}(\boldsymbol{x}, \boldsymbol{\xi})\right|_{\boldsymbol{x}=0}$ (with corresponding modifications for other variables), we have

$$
\triangle_{r p}(\boldsymbol{x}, \boldsymbol{\xi})=\triangle_{r p}(0, \boldsymbol{\xi})+\boldsymbol{x} \cdot \frac{\partial}{\partial \boldsymbol{x}} \triangle_{r p}(0, \boldsymbol{\xi})+\cdots
$$

$$
\triangle_{u p}\left(\boldsymbol{x}^{\prime}, \boldsymbol{\xi}^{\prime}\right)=\triangle_{u p}(0, \boldsymbol{\xi})+\boldsymbol{x}^{\prime} \cdot \frac{\partial}{\partial \boldsymbol{x}^{\prime}} \triangle_{u p}\left(0, \boldsymbol{\xi}^{\prime}\right)+\cdots
$$

We make the assumption that for all $r, u$, at the origin $\triangle_{r p}(0, \boldsymbol{\xi}) \approx 0$ and $\triangle_{u p}\left(0, \boldsymbol{\xi}^{\prime}\right) \approx 0$ and neglect the higher order terms in these Taylor series expansions.

By the Fourier inversion formula, we have from (11)

$$
R_{T}\left(\boldsymbol{x}, \boldsymbol{x}^{\prime}\right)=\int e^{-\mathrm{i}\left(\boldsymbol{x} \cdot \boldsymbol{\zeta}-\boldsymbol{x}^{\prime} \cdot \boldsymbol{\zeta}^{\prime}\right)} \tilde{S}_{T}\left(\boldsymbol{\zeta}, \boldsymbol{\zeta}^{\prime}\right) \mathrm{d} \boldsymbol{\zeta} \mathrm{d} \boldsymbol{\zeta}^{\prime}
$$

Therefore, we obtain

$$
\begin{aligned}
& e^{\mathrm{i}\left(\triangle_{r p}(\boldsymbol{x}, \boldsymbol{\xi})-\triangle_{u p}\left(\boldsymbol{x}^{\prime}, \boldsymbol{\xi}^{\prime}\right)\right)} R_{T}\left(\boldsymbol{x}, \boldsymbol{x}^{\prime}\right) \approx \int e^{-\mathrm{i}\left(\boldsymbol{x} \cdot \boldsymbol{\zeta}-\boldsymbol{x}^{\prime} \cdot \boldsymbol{\zeta}^{\prime}\right)} \\
& \quad \times \tilde{S}_{T}\left(\boldsymbol{\zeta}+\partial_{\boldsymbol{x}} \triangle_{r p}(0, \boldsymbol{\xi}), \boldsymbol{\zeta}^{\prime}+\partial_{\boldsymbol{x}^{\prime}} \triangle_{u p}\left(0, \boldsymbol{\xi}^{\prime}\right)\right) \mathrm{d} \boldsymbol{\zeta} \mathrm{d} \boldsymbol{\zeta}^{\prime} .
\end{aligned}
$$

Substituting this back into (94), we obtain (100), shown at the bottom of the page. We now apply the method of stationary phase to this integral. Denote the phase function

$$
\varphi_{3}\left(\boldsymbol{x}, \boldsymbol{x}^{\prime}, \boldsymbol{\xi}, \boldsymbol{\xi}^{\prime}, \boldsymbol{\zeta}, \boldsymbol{\zeta}^{\prime}\right)=(\boldsymbol{x}-\boldsymbol{z}) \cdot \boldsymbol{\xi}-\left(\boldsymbol{x}^{\prime}-z\right) \cdot \boldsymbol{\xi}^{\prime}-\boldsymbol{x} \cdot \boldsymbol{\zeta}+\boldsymbol{x}^{\prime} \cdot \boldsymbol{\zeta}^{\prime}
$$

We compute the derivatives of $\varphi_{3}$ with respect to $\boldsymbol{z}, \boldsymbol{\xi}^{\prime}, \boldsymbol{x}, \boldsymbol{\xi}$ and set it equal to 0 . We obtain the following:

$$
\xi^{\prime}=\xi, \quad \xi=\zeta, \quad z=x^{\prime}, \quad x=x^{\prime}
$$

Hence, by the method of stationary phase, we have the following integral representation [see (103), shown at the bottom of the next page]. We observe that in the integral expression (103)

$$
\begin{aligned}
I_{2} \approx & \sum_{r, u=1, r, u \neq p}^{M} \int e^{\mathrm{i}\left[(\boldsymbol{x}-\boldsymbol{z}) \cdot \boldsymbol{\xi}-\left(\boldsymbol{x}^{\prime}-z\right) \cdot \boldsymbol{\xi}^{\prime}-\left(\boldsymbol{x} \cdot \boldsymbol{\zeta}-\boldsymbol{x}^{\prime} \cdot \boldsymbol{\zeta}^{\prime}\right)\right]} \beta_{p q r}(\boldsymbol{x}, \boldsymbol{z}, \boldsymbol{\xi}) \\
& \times \overline{\beta_{p q u}\left(\boldsymbol{x}^{\prime}, \boldsymbol{z}, \boldsymbol{\xi}^{\prime}\right)} \tilde{S}_{T}\left(\boldsymbol{\zeta}+\partial_{\boldsymbol{x}} \triangle_{r p}(0, \boldsymbol{\xi}), \boldsymbol{\zeta}^{\prime}+\partial_{\boldsymbol{x}^{\prime}} \triangle_{u p}\left(0, \boldsymbol{\xi}^{\prime}\right)\right) \\
& \times \mathrm{d} \boldsymbol{x} \mathrm{d} \boldsymbol{x}^{\prime} \mathrm{d} \boldsymbol{\xi} \mathrm{d} \boldsymbol{\xi}^{\prime} \mathrm{d} \boldsymbol{z} .
\end{aligned}
$$


the leading order contribution is when $\zeta=\zeta^{\prime}$ because of the oscillatory term $e^{\mathrm{i} \boldsymbol{x}^{\prime} \cdot\left(\boldsymbol{\zeta}-\boldsymbol{\zeta}^{\prime}\right)}$. When $\boldsymbol{\zeta}=\boldsymbol{\zeta}^{\prime}$, we have

$$
\tilde{S}_{T}\left(\boldsymbol{\zeta}+\partial_{\boldsymbol{x}^{\prime}} \triangle_{r p}(0, \boldsymbol{\zeta}), \boldsymbol{\zeta}+\partial_{\boldsymbol{x}^{\prime}} \triangle_{u p}(0, \boldsymbol{\zeta})\right)=0
$$

by the stationarity assumption. Therefore, this integral expression does not have any leading order contribution to $I_{2}$. Furthermore by stationarity of $S_{T}$, we obtain

$$
\begin{array}{r}
I_{2} \approx \sum_{r=1, r \neq p}^{M} \int\left|A_{r q}\left(\boldsymbol{x}^{\prime}, \boldsymbol{\zeta}\right) B_{p q}\left(\boldsymbol{x}^{\prime}, \boldsymbol{\zeta}\right) J_{p q}\left(\boldsymbol{x}^{\prime}, \boldsymbol{x}^{\prime}, \boldsymbol{\zeta}\right)\right|^{2} \\
\times S_{T}\left(\boldsymbol{\zeta}+\partial_{\boldsymbol{x}^{\prime}} \triangle_{r p}(0, \boldsymbol{\zeta})\right) \mathrm{d} \boldsymbol{x}^{\prime} \mathrm{d} \boldsymbol{\zeta}
\end{array}
$$

\section{APPENDIX D}

Stationary Phase CALCUlation of $I_{3}$

Consider (106), shown at the bottom of the page. We compute

$$
\mathrm{E}\left[\int e^{\mathrm{i}\left(\boldsymbol{x} \cdot \boldsymbol{\zeta}-\boldsymbol{x}^{\prime} \cdot \boldsymbol{\zeta}^{\prime}\right)} T(\boldsymbol{x}) e^{-\mathrm{i} \triangle_{r p}\left(\boldsymbol{x}^{\prime}, \boldsymbol{\xi}^{\prime}\right)} T\left(\boldsymbol{x}^{\prime}\right) \mathrm{d} \boldsymbol{x} \mathrm{d} \boldsymbol{x}^{\prime}\right] \text {. }
$$

Making use of the expansion (97) and as before ignoring the first term and the higher order terms, we obtain

$$
\approx \tilde{S}_{T}\left(\boldsymbol{\zeta}, \boldsymbol{\zeta}^{\prime}+\partial_{\boldsymbol{x}^{\prime}} \triangle_{r p}\left(0, \boldsymbol{\xi}^{\prime}\right)\right)
$$

Now we have (109), shown at the bottom of the page. The phase function is the same as $\varphi_{3}$ in (101) and so the critical points are as in (102).

$$
\begin{aligned}
I_{2} & \approx \sum_{r=1, r \neq p}^{M} \int e^{\mathrm{i} \boldsymbol{x}^{\prime} \cdot\left(\boldsymbol{\zeta}^{\prime}-\boldsymbol{\zeta}\right)}\left|A_{r q}\left(\boldsymbol{x}^{\prime}, \boldsymbol{\zeta}\right) B_{p q}\left(\boldsymbol{x}^{\prime}, \boldsymbol{\zeta}\right) J_{p q}\left(\boldsymbol{x}^{\prime}, \boldsymbol{x}^{\prime}, \boldsymbol{\zeta}\right)\right|^{2} \\
& \times \tilde{S}_{T}\left(\boldsymbol{\zeta}+\partial_{\boldsymbol{x}^{\prime}} \triangle_{r p}(0, \boldsymbol{\zeta}), \boldsymbol{\zeta}^{\prime}+\partial_{\boldsymbol{x}^{\prime}} \triangle_{r p}(0, \boldsymbol{\zeta})\right) \mathrm{d} \boldsymbol{x}^{\prime} \mathrm{d} \boldsymbol{\zeta} \mathrm{d} \boldsymbol{\zeta}^{\prime} \\
& +\sum_{r, u=1, r \neq u \neq p}^{M} \int e^{\mathrm{i} \boldsymbol{x}^{\prime} \cdot\left(\boldsymbol{\zeta}^{\prime}-\boldsymbol{\zeta}\right)} A_{r q}\left(\boldsymbol{x}^{\prime}, \boldsymbol{\zeta}\right) \overline{A_{u q}\left(\boldsymbol{x}^{\prime}, \boldsymbol{\zeta}\right)}\left|J_{p q}\left(\boldsymbol{x}^{\prime}, \boldsymbol{x}^{\prime}, \boldsymbol{\zeta}\right)\right|^{2} \\
& \times\left|B_{p q}\left(\boldsymbol{x}^{\prime}, \boldsymbol{\zeta}\right)\right|^{2} \tilde{S}_{T}\left(\boldsymbol{\zeta}+\partial_{\boldsymbol{x}^{\prime}} \triangle_{r p}(0, \boldsymbol{\zeta}), \boldsymbol{\zeta}^{\prime}+\partial_{\boldsymbol{x}^{\prime}} \triangle_{u p}(0, \boldsymbol{\zeta})\right) \\
& \times \mathrm{d} \boldsymbol{x}^{\prime} \mathrm{d} \boldsymbol{\zeta} \mathrm{d} \boldsymbol{\zeta}^{\prime} .
\end{aligned}
$$

$$
\begin{aligned}
I_{3}=2 \mathrm{E}[ & \operatorname{Re} \sum_{r=1, r \neq p}^{M} \int e^{\mathrm{i}(\boldsymbol{x}-\boldsymbol{z}) \cdot \boldsymbol{\xi}-\left(\boldsymbol{x}^{\prime}-\boldsymbol{z}\right) \cdot \boldsymbol{\xi}^{\prime}} \frac{B_{p q}\left(\boldsymbol{z}, \boldsymbol{\xi}^{\prime}\right) A_{r q}\left(\boldsymbol{x}^{\prime}, \boldsymbol{\xi}^{\prime}\right)}{}\left|J_{p q}\left(\boldsymbol{x}^{\prime}, \boldsymbol{z}, \boldsymbol{\xi}^{\prime}\right)\right| \\
& \left.\times\left(B_{p q}(\boldsymbol{z}, \boldsymbol{\xi}) A_{r q}(\boldsymbol{x}, \boldsymbol{\xi})\left|J_{p q}(\boldsymbol{x}, \boldsymbol{z}, \boldsymbol{\xi})\right|-1\right) \times T(\boldsymbol{x}) \tilde{T}_{r}\left(\boldsymbol{x}^{\prime}, \boldsymbol{\xi}^{\prime}\right) \mathrm{d} \boldsymbol{x} \mathrm{d} \boldsymbol{x}^{\prime} \mathrm{d} \boldsymbol{\xi} \mathrm{d} \boldsymbol{\xi}^{\prime} \mathrm{d} \boldsymbol{z}\right] .
\end{aligned}
$$

$$
\begin{aligned}
I_{3} \approx & 2 \sum_{r=1, r \neq p}^{M} \operatorname{Re} \int e^{\mathrm{i}\left[(\boldsymbol{x}-\boldsymbol{z}) \cdot \boldsymbol{\xi}-\left(\boldsymbol{x}^{\prime}-\boldsymbol{z}\right) \cdot \boldsymbol{\xi}^{\prime}-\boldsymbol{x} \cdot \boldsymbol{\zeta}+\boldsymbol{x}^{\prime} \cdot \boldsymbol{\zeta}^{\prime}\right]} \\
& \times \overline{B_{p q}\left(\boldsymbol{z}, \boldsymbol{\xi}^{\prime}\right) A_{r q}\left(\boldsymbol{x}^{\prime}, \boldsymbol{\xi}^{\prime}\right)}\left|J_{p q}\left(\boldsymbol{x}^{\prime}, \boldsymbol{z}, \boldsymbol{\xi}^{\prime}\right)\right| \\
& \times\left(B_{p q}(\boldsymbol{z}, \boldsymbol{\xi}) A_{r q}(\boldsymbol{x}, \boldsymbol{\xi})\left|J_{p q}(\boldsymbol{x}, \boldsymbol{z}, \boldsymbol{\xi})\right|-1\right) \\
& \times \tilde{S}_{T}\left(\boldsymbol{\zeta}, \boldsymbol{\zeta}^{\prime}+\partial_{\boldsymbol{x}^{\prime}} \triangle_{r p}\left(0, \boldsymbol{\xi}^{\prime}\right)\right) \mathrm{d} \boldsymbol{x} \mathrm{d} \boldsymbol{x}^{\prime} \mathrm{d} \boldsymbol{\xi} \mathrm{d} \boldsymbol{\xi}^{\prime} \mathrm{d} \boldsymbol{\zeta} \mathrm{d} \boldsymbol{\zeta}^{\prime} \mathrm{d} \boldsymbol{z} .
\end{aligned}
$$

$$
\begin{aligned}
& I_{3} \approx 2 \sum_{r=1, r \neq p}^{M} \operatorname{Re} \int e^{\mathrm{i} \boldsymbol{x}^{\prime} \cdot\left(\boldsymbol{\zeta}^{\prime}-\boldsymbol{\zeta}\right)} \overline{B_{p q}\left(\boldsymbol{x}^{\prime}, \boldsymbol{\zeta}\right) A_{r q}\left(\boldsymbol{x}^{\prime}, \boldsymbol{\zeta}\right)} \\
& \times\left(B_{p q}\left(\boldsymbol{x}^{\prime}, \boldsymbol{\zeta}\right) A_{r q}\left(\boldsymbol{x}^{\prime}, \boldsymbol{\zeta}\right)\left|J_{p q}\left(\boldsymbol{x}^{\prime}, \boldsymbol{z}, \boldsymbol{\xi}^{\prime}\right)\right|-1\right) \\
& \times\left|J_{p q}\left(\boldsymbol{x}^{\prime}, \boldsymbol{x}^{\prime}, \boldsymbol{\zeta}\right)\right| \tilde{S}_{T}\left(\boldsymbol{\zeta}, \boldsymbol{\zeta}^{\prime}+\partial_{\boldsymbol{x}^{\prime}} \triangle_{r p}(0, \boldsymbol{\zeta})\right) \mathrm{d} \boldsymbol{x}^{\prime} \mathrm{d} \boldsymbol{\zeta} \mathrm{d} \boldsymbol{\zeta}^{\prime} .
\end{aligned}
$$


Applying the method of stationary phase, we obtain (110), shown at the bottom of the previous page. The leading order contribution to this integral is when $\boldsymbol{\zeta}=\boldsymbol{\zeta}^{\prime}$ because of the oscillatory term $e^{\mathrm{i} \boldsymbol{x}^{\prime} \cdot\left(\boldsymbol{\zeta}^{\prime}-\boldsymbol{\zeta}\right)}$ in the integral. Hence, we have the term $\left.\tilde{S}_{T}\left(\boldsymbol{\zeta}, \boldsymbol{\zeta}+\partial_{\boldsymbol{x}^{\prime}} \triangle_{r p}(0, \boldsymbol{\zeta})\right)\right)$ in the integrand and assuming stationarity of $\tilde{S}_{T}$, this is 0 . Therefore, there is no leading order contribution of this integral to the determination of the filter $B_{p q}$.

\section{APPENDIX E}

\section{VARIATIONAL DERIVATIVE}

We have $I\left(B_{p q}\right)=I_{1}+I_{2}+I_{4}$. Let us write (111), shown at the bottom of the page. Fix a function $B_{0}\left(\boldsymbol{x}^{\prime}, \boldsymbol{\zeta}\right)$ and for small $\varepsilon$ consider a variation of the form $B_{\varepsilon}=B_{p q}+\varepsilon B_{0}$. We substitute this into the above equation and differentiate with respect to $\varepsilon$ and then set $\varepsilon=0$. We have (112), shown at the bottom of the page. We differentiate $I\left(B_{p q}+\varepsilon B_{0}\right)$ with respect to $\varepsilon$ and set $\varepsilon=0$. We obtain (113), shown at the bottom of the page. Since this is 0 for all $B_{0}$, we have (114), shown at the bottom of the page. Canceling out the Jacobian term and rearranging, we obtain

$$
B_{p q}\left(\boldsymbol{x}^{\prime}, \boldsymbol{\zeta}\right)=\frac{\overline{A_{p q}\left(\boldsymbol{x}^{\prime}, \boldsymbol{\zeta}\right)} S_{T}(\boldsymbol{\zeta})}{\left|J_{p q}\left(\boldsymbol{x}^{\prime}, \boldsymbol{x}^{\prime}, \boldsymbol{\zeta}\right)\right| \times \Psi+S_{n}(\boldsymbol{\zeta})}
$$

where

$$
\begin{aligned}
\Psi=\mid & \left.A_{p q}\left(\boldsymbol{x}^{\prime}, \boldsymbol{\zeta}\right)\right|^{2} S_{T}(\boldsymbol{\zeta}) \\
& +\sum_{r=1, r \neq p}^{M}\left|A_{r q}\left(\boldsymbol{x}^{\prime}, \boldsymbol{\zeta}\right)\right|^{2} S_{T}\left(\boldsymbol{\zeta}+\partial_{\boldsymbol{x}^{\prime}} \triangle_{r p}(0, \boldsymbol{\zeta})\right)
\end{aligned}
$$

$$
\begin{aligned}
I\left(B_{p q}\right)= & \int\left|B_{p q}(\boldsymbol{x}, \boldsymbol{\zeta}) A_{p q}(\boldsymbol{x}, \boldsymbol{\zeta})\right| J_{p q}(\boldsymbol{x}, \boldsymbol{x}, \boldsymbol{\zeta})|-1|^{2} \times S_{T}(\boldsymbol{\zeta}) \mathrm{d} x \mathrm{~d} \boldsymbol{\zeta} \\
& +\sum_{r=1, r \neq p}^{M} \int\left|A_{r q}(\boldsymbol{x}, \boldsymbol{\zeta}) B_{p q}(\boldsymbol{x}, \boldsymbol{\zeta}) J_{p q}(\boldsymbol{x}, \boldsymbol{x}, \boldsymbol{\zeta})\right|^{2} \times S_{T}\left(\boldsymbol{\zeta}+\partial_{\boldsymbol{x}} \triangle_{r p}(0, \boldsymbol{\zeta})\right) \mathrm{d} \boldsymbol{x} \mathrm{d} \boldsymbol{\zeta} \\
& +\int\left|B_{p q}(\boldsymbol{x}, \boldsymbol{\zeta})\right|^{2} S_{n}(\boldsymbol{\zeta})|J(\boldsymbol{x}, \boldsymbol{x}, \boldsymbol{\zeta})| \mathrm{d} \boldsymbol{x} \mathrm{d} \boldsymbol{\zeta} .
\end{aligned}
$$

$$
\begin{aligned}
I\left(B_{p q}+\varepsilon B_{0}\right)= & \int\left|\left(B_{p q}+\varepsilon B_{0}\right)\left(\boldsymbol{x}^{\prime}, \boldsymbol{\zeta}\right) A_{p q}\left(\boldsymbol{x}^{\prime}, \boldsymbol{\zeta}\right)\right| J_{p q}\left(\boldsymbol{x}^{\prime}, \boldsymbol{x}^{\prime}, \boldsymbol{\zeta}\right)|-1|^{2} \times S_{T}(\boldsymbol{\zeta}) \mathrm{d} x^{\prime} \mathrm{d} \boldsymbol{\zeta} \\
& +\sum_{r=1, r \neq p}^{M} \int\left|A_{r q}\left(\boldsymbol{x}^{\prime}, \boldsymbol{\zeta}\right)\left(B_{p q}+\varepsilon B_{0}\right)\left(\boldsymbol{x}^{\prime}, \boldsymbol{\zeta}\right) J_{p q}\left(\boldsymbol{x}^{\prime}, \boldsymbol{x}^{\prime}, \boldsymbol{\zeta}\right)\right|^{2} \times S_{T}\left(\boldsymbol{\zeta}+\partial_{\boldsymbol{x}^{\prime}} \triangle_{r p}(0, \boldsymbol{\zeta})\right) \mathrm{d} \boldsymbol{x}^{\prime} \mathrm{d} \boldsymbol{\zeta} \\
& +\int\left|\left(B_{p q}+\varepsilon B_{0}\right)\left(\boldsymbol{x}^{\prime}, \boldsymbol{\zeta}\right)\right|^{2} S_{n}(\boldsymbol{\zeta})\left|J\left(\boldsymbol{x}^{\prime}, \boldsymbol{x}^{\prime}, \boldsymbol{\zeta}\right)\right| \mathrm{d} \boldsymbol{x}^{\prime} \mathrm{d} \boldsymbol{\zeta} .
\end{aligned}
$$

$$
\begin{aligned}
0= & 2 \operatorname{Re} \int\left(B_{p q}\left(\boldsymbol{x}^{\prime}, \boldsymbol{\zeta}\right) A_{p q}\left(\boldsymbol{x}^{\prime}, \boldsymbol{\zeta}\right)\left|J_{p q}\left(\boldsymbol{x}^{\prime}, \boldsymbol{x}^{\prime}, \boldsymbol{\zeta}\right)\right|-1\right) \times \overline{B_{0}\left(\boldsymbol{x}^{\prime}, \boldsymbol{\zeta}\right) A_{p q}\left(\boldsymbol{x}^{\prime}, \boldsymbol{\zeta}\right)\left|J_{p q}\left(\boldsymbol{x}^{\prime}, \boldsymbol{x}^{\prime}, \boldsymbol{\zeta}\right)\right|} S_{T}(\boldsymbol{\zeta}) \mathrm{d} \boldsymbol{x}^{\prime} \mathrm{d} \boldsymbol{\zeta} \\
& +\sum_{r=1, r \neq p}^{M} 2 \operatorname{Re} \int B_{p q}\left(\boldsymbol{x}^{\prime}, \boldsymbol{\zeta}\right) \overline{B_{0}\left(\boldsymbol{x}^{\prime}, \boldsymbol{\zeta}\right)}\left|A_{r q}\left(\boldsymbol{x}^{\prime}, \boldsymbol{\zeta}\right) J_{p q}\left(\boldsymbol{x}^{\prime}, \boldsymbol{x}^{\prime}, \boldsymbol{\zeta}\right)\right|^{2} \times \tilde{S}_{T}\left(\boldsymbol{\zeta}+\partial_{\boldsymbol{x}^{\prime}} \triangle_{r p}(0, \boldsymbol{\zeta}), \boldsymbol{\zeta}^{\prime}+\partial_{\boldsymbol{x}^{\prime}} \triangle_{r p}(0, \boldsymbol{\zeta})\right) \mathrm{d} \boldsymbol{x}^{\prime} \mathrm{d} \boldsymbol{\zeta} \mathrm{d} \boldsymbol{\zeta}^{\prime} \\
& +2 \operatorname{Re} \int B_{p q}\left(\boldsymbol{x}^{\prime}, \boldsymbol{\zeta}\right) \overline{B_{0}\left(\boldsymbol{x}^{\prime}, \boldsymbol{\zeta}\right)} S_{n}(\boldsymbol{\zeta})\left|J\left(\boldsymbol{x}^{\prime}, \boldsymbol{x}^{\prime}, \boldsymbol{\zeta}\right)\right| \mathrm{d} \boldsymbol{x}^{\prime} \mathrm{d} \boldsymbol{\zeta}
\end{aligned}
$$

$$
\begin{aligned}
0= & \left(B_{p q}\left(\boldsymbol{x}^{\prime}, \boldsymbol{\zeta}\right) A_{p q}\left(\boldsymbol{x}^{\prime}, \boldsymbol{\zeta}\right)\left|J_{p q}\left(\boldsymbol{x}^{\prime}, \boldsymbol{x}^{\prime}, \boldsymbol{\zeta}\right)\right|-1\right) \times \overline{A_{p q}\left(\boldsymbol{x}^{\prime}, \boldsymbol{\zeta}\right)}\left|J_{p q}\left(\boldsymbol{x}^{\prime}, \boldsymbol{x}^{\prime}, \boldsymbol{\zeta}\right)\right| S_{T}(\boldsymbol{\zeta}) \\
& +\sum_{r=1, r \neq p}^{M} B_{p q}\left(\boldsymbol{x}^{\prime}, \boldsymbol{\zeta}\right)\left|A_{r q}\left(\boldsymbol{x}^{\prime}, \boldsymbol{\zeta}\right) J_{p q}\left(\boldsymbol{x}^{\prime}, \boldsymbol{x}^{\prime}, \boldsymbol{\zeta}\right)\right|^{2} \times S_{T}\left(\boldsymbol{\zeta}+\partial_{\boldsymbol{x}^{\prime}} \triangle_{r p}(0, \boldsymbol{\zeta})\right) \\
& +B_{p q}\left(\boldsymbol{x}^{\prime}, \boldsymbol{\zeta}\right) S_{n}(\boldsymbol{\zeta})\left|J\left(\boldsymbol{x}^{\prime}, \boldsymbol{x}^{\prime}, \boldsymbol{\zeta}\right)\right| .
\end{aligned}
$$


Taking into account the data collection manifold (26) and putting a characteristic function cut-off for those $\boldsymbol{\zeta}$ that belongs to this set, we arrive at (56).

\section{ACKNOWLEDGMENT}

The authors would like to thank Prof. B. Rigling for his valuable comments, especially for his suggestions regarding the content of Section V. J. Swoboda's affiliation with the MITRE Corporation is provided for identification purposes only and is not intended to convey or imply MITRE's concurrence with, or support for, the positions, opinions, or viewpoints expressed by the author.

\section{REFERENCES}

[1] L.-E. Andersson, "On the determination of a function from spherical averages," SIAM J. Math. Anal., vol. 19, no. 1, pp. 214-232, 1988.

[2] O. Arikan and D. C. Munson, Jr, "A tomographic formulation of bistatic synthetic aperture radar," Proc. ComCon, p. 418, Oct. 1988.

[3] I. Bradaric, G. T. Capraro, D. D. Weiner, and M. C. Wicks, "A framework for the analysis of multistatic radar systems with multiple transmitters," in Proc. Electromagnetics in Advanced Applications, 2007, pp. 443-446.

[4] I. Bradaric, G. T. Capraro, and M. C. Wicks, "Waveform diversity for different multistatic radar configurations," in Proc. 41st Asilomar Conf. Signals, Systems and Computers, 2007, pp. 2038-2042.

[5] M. Cheney, "Synthetic-aperture assessment of a dispersive surface," Int. J. Imag. Syst. Technol., vol. 14, pp. 28-34, 2004.

[6] M. Cheney and B. Yazici, "Radar imaging with independently moving transmitters and receivers," presented at the Defense Advance Signal Processing (DASP) Workshop, Dec. 2006.

[7] S. R. Doughty, K. Woodbridge, and C. J. Baker, "Improving resolution using multistatic radar," in Proc. IET Int. Conf. Radar Systems, 2007, pp. $1-5$.

[8] J. J. Duistermaat, Fourier Integral Operators, Progress in Mathematics. Boston, MA: Birkhäuser, 1996, vol. 130.

[9] W. Goj, Synthetic Aperture Radar and Electronic Warfare. Boston, MA: Artech House, 1993.

[10] A. Grigis and J. Sjöstrand, Microlocal Analysis for Differential Operators, An Introduction. Cambridge, U.K.: Cambridge Univ. Press, 1994, vol. 196.

[11] V. Guillemin and S. Sternberg, Geometric Asymptotics. Providence, R.I: American Mathematical Society, 1977.

[12] M. Gustafsson, Multi-Static Synthetic Aperture Radar and Inverse Scattering 2004, Tech. Rep. LUTEDX/(TEAT-7123)/1-28/(2003).

[13] A. M. Horne and G. Yates, "Bistatic synthetic aperture radar," in Proc. IEEE Radar Conf., Oct. 2002, pp. 6-10.

[14] L. Ibanez and W. Schroeder, The Insight Toolkit (ITK) Software Guide 2.4. New York: Kitware, Inc., 787 pp., Nov. 21, 2005.

[15] G. Krieger and A. Moreira, "Spaceborne bi- and multistatic SAR: Potential and challenges," IEE Proc. Radar, Sonar and Navigation, vol. 153, no. 3, pp. 184-198, 2006

[16] G. Krieger and M. Younis, "Impact of oscillator noise in bistatic and multistatic SAR," IEEE Geosc. Remote Sens. Lett., vol. 3, no. 3, pp. 424-428, 2006.

[17] K.-H. Liu and D. C. Munson, "Autofocus in multistatic passive SAR imaging," in Proc. IEEE Int. Conf. Acoustics, Speech and Signal Processing, 2008, pp. 1277-1280.

[18] R. L. Moses and L. C. Potter, "Noncoherent 2D and 3D SAR reconstruction from wide-angle measurements," presented at the 13th Annu. Adaptive Sensor Array Processing Workshop, Lexington, MA, Jun. 7-8, 2005.

[19] S. Nilsson, "Application of Fast Backprojection Techniques for Some Inverse Problems of Integral Geometry," Ph.D. dissertation, Linköping Studies Sci.Technol., Linköping, 1997.

[20] C. J. Nolan and M. Cheney, "Synthetic aperture inversion," Inv. Probl., vol. 18, pp. 221-236, 2002.

[21] C. J. Nolan and M. Cheney, "Synthetic aperture inversion for arbitrary flight paths and non-flat topography," IEEE Trans. Image Process., vol. 12, pp. 1035-1043, 2003.
[22] C. J. Nolan and M. Cheney, "Microlocal analysis of synthetic aperture radar imaging," J. Fourier Anal. Appl., vol. 10, pp. 133-148, 2004.

[23] B. Rigling, "Signal Processing Strategies for Bistatic Synthetic Aperture Radar," Ph.D. dissertation, The Ohio State Univ., Columbus, 2003.

[24] B. D. Rigling and R. L. Moses, "Three-dimensional surface reconstruction from multistatic SAR images," IEEE Trans. Image Process., vol. 14, pp. 1159-1171, 2005.

[25] M. Soumekh, "Bistatic synthetic aperture radar inversion with application in dynamic object imaging," IEEE Trans. Signal Process., vol. 39, pp. 2044-2055, 1991.

[26] M. Soumekh, "Wide-bandwidth continuous-wave monostatic and bistatic synthetic aperture radar imaging," in Proc. Int. Conf. Image Processing, Oct. 1998, vol. 3, pp. 361-365.

[27] J. Swoboda, C. E. Yarman, and B. Yazici, "Bistatic synthetic aperture radar imaging for arbitrary trajectories in the presence of noise and clutter," presented at the SPIE Defense and Security Conf, Apr. 2009.

[28] T. Teer and N. A. Goodman, "Multistatic SAR algorithm with image combination," in Proc. IEEE Conf. Radar, 2006, p. 8

[29] F. Trèves, Introduction to Pseudodifferential and Fourier Integral Operators. New York: Plenum, 1980, vol. 1.

[30] F. Trèves, Introduction to Pseudodifferential and Fourier Integral Operators. New York: Plenum, 1980, vol. 2.

[31] L. M. H. Ulander and T. Martin, "Bistatic ultrawideband SAR for imaging of ground targets under foliage," in Proc. IEEE Radar Conf., May 2005, pp. 419-423.

[32] N. J. Willis, Bistatic Radar. Norwood, MA: Artech House, 1991.

[33] C. E. Yarman, B. Yazici, and M. Cheney, "Bistatic synthetic aperture radar imaging for arbitrary flight trajectories," IEEE Trans. Image Process., vol. 17, pp. 84-93, 2008.

[34] B. Yazici, M. Cheney, and C. E. Yarman, "Synthetic-aperture inversion in the presence of noise and clutter," Inv. Probl., vol. 22, pp. 1705-1729, 2006.

V. Krishnan received the M.S. degree in mathematics from Pondicherry University, India, and the Ph.D. degree in mathematics from the University of Washington, Seattle, in 2007.

$\mathrm{He}$ is a postdoctoral research associate with the Department of Electrical, Computer and Systems Engineering at Rensselaer Polytechnic Institute, Troy, NY. His main research interest is in inverse problems.

J. Swoboda (M'07) received the B.S. degree in electrical and computer systems engineering and the M.S. degree in electrical engineering from Rensselaer Polytechnic Institute, Troy NY, in 2007 and 2008, respectively.

He is currently with the MITRE Corporation, Bedford, MA, focusing primarily in the areas of signal processing and radar systems.

C. E. Yarman (M'07) received the B.Sc. degree in mathematics from the Middle East Technical University, Ankara, Turkey, and the M.Sc. degree in biomedical science from Drexel University, Philadelphia, PA, and the M.Sc. degree in mathematics and the Ph.D. in electrical engineering from Rensselaer Polytechnic Institute (RPI), Troy, NY, in 2006.

He was a postdoctoral research associate at RPI from May 2006 to February 2007. Currently, he is a Research Scientist at the Houston Technology Center, WesternGeco-Schlumberger, Houston, TX. His main research interest is inverse problems in imaging.

B. Yazici (SM'06) received the B.S. degrees in electrical engineering and mathematics from Bogazici University, Istanbul, Turkey, in 1988, and the M.S. and $\mathrm{Ph} . \mathrm{D}$. degrees in mathematics and electrical engineering from Purdue University, West Lafayette IN, in 1990 and 1994, respectively.

From September 1994 until 2000, she was a Research Engineer at the General Electric Company Global Research Center, Schenectady, NY. During her tenure in industry, she worked on radar, transportation, industrial, and medical imaging systems. In 2003, she joined Rensselaer Polytechnic Institute, Troy, NY, where she is currently an Associate Professor in the Department of Electrical, Computer, and Systems Engineering and in the Department of Biomedical Engineering. Her research interests span the areas of statistical signal processing, inverse problems in imaging, biomedical optics, and radar. She holds 11 U.S. patents.

Dr. Yazici is the recipient of the Rensselaer Polytechnic Institute 2007 School of Engineering Research Excellence Award. Her work on industrial systems received the second best paper award in 1997 given by IEEE TRANSACTIONS ON INDUSTRIAL APPLICATIONS. 